\title{
Generalized incremental frequency method for topological design of continuum structures for minimum dynamic compliance subject to forced vibration at a prescribed low or high value of the excitation frequency
}

\author{
Niels Olhoff ${ }^{1} \cdot$ Jianbin $\mathrm{Du}^{2}$
}

Received: 31 December 2015 /Revised: 16 August 2016 / Accepted: 18 August 2016/Published online: 15 September 2016

(C) The Author(s) 2016. This article is published with open access at Springerlink.com

\begin{abstract}
This paper deals with topological design optimization of elastic, continuum structures without damping that are subjected to time-harmonic, design-independent external dynamic loading with prescribed excitation frequency, amplitude and spatial distribution. The admissible design domain, the boundary conditions, and the available amount(s) of material(s) for single- or bi-material structures are given. An important objective of such a design problem is often to drive the resonance frequencies of the structure as far away as possible from the given excitation frequency in order to avoid resonance and to reduce the vibration level of the structure. In the present paper, the excitation frequency is defined to be 'low' for positive values up to and including the fundamental resonance frequency of the structure, and to be 'high' for values beyond that. Our paper shows that it may be very important to consider different design paths in problems of minimum dynamic compliance in order to obtain desirable solutions for prescribed excitation frequencies, and a socalled 'incremental frequency technique' (IF technique) is
\end{abstract}

This paper is dedicated to the memory of Professor George Rozvany, please refer to the Acknowledgement.

Electronic supplementary material The online version of this article (doi:10.1007/s00158-016-1574-3) contains supplementary material, which is available to authorized users.

Jianbin Du

dujb@tsinghua.edu.cn

Niels Olhoff

no@m-tech.aau.dk

1 Department of Mechanical and Manufacturing Engineering, Aalborg University, DK-9220 Aalborg East, Denmark

2 School of Aerospace Engineering, Tsinghua University, Beijing 100084, People's Republic of China applied for this. Subsequently, the IF technique is integrated into an extended, systematic method named as the 'generalized incremental frequency method' (GIF method) that is developed for gradient based dynamic compliance minimization for not only 'low', but also 'high' excitation frequencies of the structure. The GIF method performs search for and determination of the solution to the minimum dynamic compliance design problem, but this is subject to the complexity that problems with prescribed high excitation frequencies exhibit disjointed design sub-spaces. Each of these sub-spaces is associated with a local minimum value of the dynamic compliance, so in general the 'global optimum solution' will have to be selected as the 'best' solution from among a number of local candidate solutions. Illustrative examples of application of the IF technique and the GIF method are presented in the paper.

Keywords Topology optimization · Continuum structures · Minimum dynamic compliance $\cdot$ Harmonic vibrations . Low and high excitation frequencies $\cdot$ IF technique . GIF method

\section{Introduction}

Since the original papers (Bendsøe and Kikuchi 1988; Bendsøe 1989), topology optimization of continuum structures has manifested itself as an extremely active area of research as reflected in the more recent publications like the review and survey papers (Eschenauer and Olhoff 2001; Kang et al. 2006; Deaton and Grandhi 2014), the textbook (Bendsøe and Sigmund 2003), and the proceedings (Bendsøe et al. 2006; Rozvany and Lewinski 2013), where new areas of application of topology optimization are discussed, and an abundance of references are available. 
Thus, among several other sub-areas, during the latest decades, topology optimization of continuum structures has been developed and applied in the following sub-areas of dynamic and vibro-acoustic topological design,

- dynamic compliance and response (Ma et al. 1995; Min et al. 1999; Shu et al. 2011; Kang et al. 2012; Yang and Li 2013; Zhang and Kang 2014; Zhao and Wang 2015; Xu et al. 2015; Jung et al. 2015; Liu et al. 2015; Olhoff and Niu 2015),

- vibro-acoustic response (Jog 2002a, b, Olhoff and Du 2006; Jensen 2007; Calvel and Mongeau 2007; Du and Olhoff 2007a; Niu et al. 2010; Yoon 2010; Nandy and Jog 2011; Du et al. 2011; Yang and Du 2012; Du and Yang 2015), and

- eigenfrequencies, eigenfrequency gaps, and band-gaps (Sigmund 2001; Sigmund and Jensen 2003; Jensen 2003; Halkjær et al. 2006; Du and Olhoff 2007a, b, c, Olhoff et al. 2012).

To the knowledge of the authors, it seems that in previous works on dynamic compliance and vibro-acoustic topology design optimization, only little attention has been paid to the difficulty that for a prescribed 'high' excitation frequency of forced vibration, a gradient-based optimization algorithm (like e.g., the MMA method, Svanberg 1987) will normally only furnish a local optimum solution. This is due to the multiple non-convexity of the response curve for the dynamic compliance depicted in Fig. 4, and is briefly explained below. According to our experiences and studies, it is necessary to overcome this difficulty because a local optimum may often be located far away from the 'global' optimum and be useless in engineering practice, so it is important to address this problem.

It is important to emphasize that in the context of this paper, the 'fundamental (first) resonance frequency' denotes the lowest eigenfrequency of vibration whose eigenmode is not orthogonal to the amplitude mode of the prescribed excitation.

Moreover, the term 'low' excitation frequencies covers given excitation (i.e. loading) frequencies with positive values up to and including the fundamental resonance frequency (or lowest / first eigenfrequency of vibration) of the structure. All other positive excitation frequencies are labelled as 'high' excitation frequencies. This implies that the upper limit for the 'low' excitation frequencies is independent of the level of the external excitation frequency, and hence constitutes a more objective limit for minimum dynamic compliance design.

An important incremental technique called the 'incremental frequency technique' (IF technique) was used very successfully for the handling of the optimum design problems, because, both for low and high excitation frequencies, this technique can be applied to sequentially increase (or decrease) a variable excitation frequency from a suitably chosen initial value up to (or down to) a prescribed, fixed value of the excitation frequency. At the same time, the IF technique enables the variable excitation frequency to 'push' a resonance frequency of given order up (or down) in the frequency response diagram.

Subsequently, the IF technique was integrated into a systematic, generalized method termed the 'generalized incremental frequency method' (GIF method) which we developed for search and determination of the topological design that minimizes the dynamic compliance for a fixed, prescribed low or high value of the excitation frequency.

With the GIF method and its governing equations for optimality at hand, a prescribed 'low' excitation frequency relative to the initial design will be also low in the optimized design (i.e, less than or equal to the fundamental resonance frequency of the optimized structure). This means that a unique, possibly global optimum solution can be determined subject to a prescribed low excitation frequency.

The situation is more complex in cases where a fixed, prescribed high excitation frequency is specified for the optimization. The reason is that the prescribed high excitation frequency implies that the optimization problem is characterized by having a number of disjointed design sub-intervals, see Fig. 4, such that it is necessary to consider the possibility that there may exist a set of different local optimum designs for which the prescribed excitation frequency is placed between different pairs of consecutive resonance frequencies. These resonance frequencies are high, and their orders are different from those of the initial design. Using the IF technique, we finally identify the local optimum design that is associated with the smallest value of dynamic compliance obtained, and hereby determine the design that may be considered as the global optimum solution to the problem with a high excitation frequency.

It is believed that in addition to specific minimum dynamic compliance design problems for macro-structures, the GIF method may also be applied to general vibro-acoustic design problems with both macro-level and micro-level.

The current paper is organized as follows. Section 2 first presents the conventional mathematical formulation of a single- or bi-material topology optimization problem subject to a prescribed excitation frequency. This is followed in Section 2.1 by brief descriptions of the SIMP interpolation models for design of continuum structures with single- and bi-materials, and Section 2.2 discusses simple modifications of these models that may hinder occurrence of spurious localized vibration modes. Section 2.3 then presents the adjoint sensitivity analysis of the objective function of the optimization problem, covering both the cases of design-independent and design-dependent loads. In Section 2.4, the very useful 'incremental frequency technique' (IF technique) that is applicable for both problems with low and high prescribed excitation frequencies, is defined. Section 2.5 discusses the basic 
features of the IF technique based topology design of singlematerial structures for minimum dynamic compliance subject to a 'high' and a 'low' excitation frequency, respectively.

Section 3 presents some numerical examples of the IF technique based topology optimization of single-material structures. Two different design paths are investigated for the design of a plate-like, single-material structure in two subexamples with a prescribed 'low' excitation frequency. Here, the first path leads to a 'degenerate' design with a very large value of the static compliance, while the second path furnishes a very desirable design with very low dynamic compliance and a much improved static compliance.

Section 4 presents in detail the development of the GIF method that is based on the IF technique described in Section 2.4, and a procedure and a flow chart of the GIF method are developed and discussed in Sections 4.1, 4.1.1 and 4.1.2. The procedure is a single, unified algorithm for handling of both 'low' and 'high' excitation frequencies, as well as single- and bi-material structures. In Section 4.1.1, this algorithm is extended to be able to solve design problems with a static compliance constraint as well.

In Section 5, two numerical examples demonstrate the usefulness of the GIF method for obtaining the 'global optimum' for a minimum dynamic compliance design problem associated with a prescribed 'high' value of the external excitation frequency.

Finally, Section 6 provides a summary of the paper.

\section{Formulation of topology design problem for minimization of dynamic compliance}

A 'conventional formulation' of the problem of optimizing the topology of a continuum structure without damping for minimum value of the integral dynamic compliance of a single- or bi-material structure can be written as follows in a finite element setting:

$$
\begin{aligned}
& \min _{\rho_{e}}\left\{F=C_{d}^{2}\right\} \\
& \text { Subject to : } \\
& \quad C_{d}=\left|\mathbf{P}^{\mathbf{T}} \mathbf{U}\right|, \\
& \left(\mathbf{K}-\omega^{2} \mathbf{M}\right) \mathbf{U}=\mathbf{P}, \\
& \sum_{e=1}^{N_{E}} \rho_{e} V_{e}-V^{*} \leq 0, \quad\left(V^{*}=\alpha V_{0}\right), \\
& 0<\underline{\rho} \leq \rho_{e} \leq 1, \quad\left(e=1, \cdots, N_{E}\right) .
\end{aligned}
$$

In (1a,b), the symbol $C_{d}$ stands for the dynamic compliance defined as $C_{d}=\left|\mathbf{P}^{\mathbf{T}} \mathbf{U}\right|$. Here, $\mathbf{P}$ denotes the vector of amplitudes of a given external time-harmonic mechanical surface loading vector $\mathbf{p}(t)=\mathbf{P} e^{i \omega t}$, where $\omega$ denotes an external excitation frequency that may be assigned the prescribed fixed value $\omega_{p}$, and the symbol $\mathbf{U}$ in $(1 \mathrm{~b}, \mathrm{c})$ represents the vector of magnitudes of the corresponding structural displacement response vector $\mathbf{a}(t)=\mathbf{U} e^{i \omega t}$. Thus, $\mathbf{U}$ and $\mathbf{P}$ satisfy the dynamic equilibrium equation (1c) for the steady-state harmonic vibration at the external frequency $\omega$, with $\mathbf{K}$ and $\mathbf{M}$ representing the $N$ dimensional global structural stiffness and mass matrices of the structure, where $N$ is the number of DOFs. We note that the above expression for the dynamic compliance $C_{d}$ represents the numerical mean value of the magnitudes of the surface displacements weighted by the values of the amplitudes of the corresponding time-harmonic surface loading. For the case of static loading $(\omega=0)$, the expression directly reduces to the traditional definition of static compliance, i.e., the work done by the external forces against corresponding displacements at equilibrium.

In (1d), $N_{E}$ denotes the total number of finite elements in the admissible design domain for the topology optimization problem. The symbols $\rho_{\mathrm{e}}, e=1, \ldots, N_{E}$, play the role as design variables of the problem and represent the volumetric material densities of the finite elements, with lower and upper limits $\underline{\rho}$ and 1 specified for $\rho_{\mathrm{e}}$ in (1e). To avoid singularity of the stiffness matrix, $\rho$ is not zero, but taken to be a small positive value like $\rho=10^{-3}$. In the volume constraint (1d), the symbol $\alpha$ defines the volume fraction $V^{*} / V_{0}$, where $V_{0}$ is the volume of the admissible design domain, and $V^{*}$ is the given available volume, respectively, of the solid material for a single-material design problem and of the stiffer material 1 * for a bi-material problem.

It is noted from (1c) that the global dynamic stiffness matrix $\mathbf{K}_{d}$ defined as $\mathbf{K}_{d}=\mathbf{K}-\omega_{p}^{2} \mathbf{M}$ may be negative definite when the prescribed external excitation frequency $\omega_{p}$ has a high value, i.e., higher than the fundamental eigenfrequency of free vibrations of the structure. In this case, we may have $\mathbf{P}^{T} \mathbf{U}<0$, and in order to include this possibility in our problem formulation, we apply the absolute value of this scalar product as the dynamic compliance $C_{d}$, see (1b). Moreover, to render the problem differentiable, we choose the objective function $F$ as the square of the dynamic compliance. As indicated by 1 , the optimization problem is written in a 'nested' formulation, and it is solved by successive iterations. In each iteration, the dynamic equilibrium equation (1c) is solved in a direct way by Gauss elimination in this paper.

\subsection{SIMP interpolation models for topology optimization}

\subsubsection{SIMP model for single-material structures}

The SIMP (Solid Isotropic Microstructure with Penalty) interpolation model for single-material topology design (see, e.g., Bendsøe 1989; Rozvany et al. 1992; Rozvany and Zhou 1991; Bendsøe and Sigmund 1999) is used together with a density filtering technique, see (Sigmund 1997; Sigmund and 
Petersson 1998), or (Bourdin 2001; Bruns and Tortorelli 2001; Sigmund 2007), in order to avoid checkerboard formation and dependency of topology solutions on finite element mesh-refinement. Thus, the finite element elasticity matrix $\mathbf{E}_{e}$ is expressed in terms of the elemental volumetric material density $\rho_{e}, 0 \leq \rho_{e} \leq 1$, and the penalization power $p, p \geq 1$, as

$$
\mathbf{E}_{e}\left(\rho_{e}\right)=\rho_{e}^{p} \mathbf{E}_{e}^{*}
$$

where $\mathbf{E}_{e}^{*}$ is the elasticity matrix of a corresponding element with the fully solid elastic material the structure is to be made of. By analogy with (2), for a vibrating structure the finite element mass matrix may be expressed as

$\mathbf{M}_{e}\left(\rho_{e}\right)=\rho_{e}^{q} \mathbf{M}_{e}^{*}$

where $\mathbf{M}_{e}^{*}$ represents the elemental mass matrix corresponding to fully solid material, and the power $q \geq 1$. Apart from exceptions briefly discussed in Section 2.2, normally $q=1$ is chosen. The global stiffness matrix $\mathbf{K}$ and mass matrix $\mathbf{M}$ for the finite element based structural response analyses behind the optimization can now be calculated by

$\mathbf{K}=\sum_{e=1}^{N_{E}} \rho_{e}^{p} \mathbf{K}_{e}^{*}, \quad \mathbf{M}=\sum_{e=1}^{N_{E}} \rho_{e}^{q} \mathbf{M}_{e}^{*}$

where $\mathbf{K}_{e}^{*}$ is the stiffness matrix of a finite element with fully solid material, and $N_{E}$ denotes the total number of finite elements in the admissible design domain.

\subsubsection{Extended SIMP model for bi-material structures}

The SIMP model for single-material design discussed in Section 2.1.1 can be easily extended to bi-material design by using the rule of mixtures (Bendsøe and Sigmund 1999; Du and Olhoff 2007a,b). The finite element elasticity matrix for the bi-material problem may be expressed as

$\mathbf{E}_{e}\left(\rho_{e}\right)=\rho_{e}^{r} \mathbf{E}_{e}^{* 1}+\left(1-\rho_{e}^{r}\right) \mathbf{E}_{e}^{* 2}$

where $\mathbf{E}_{e}^{* 1}$ and $\mathbf{E}_{e}^{* 2}$ denote the element elasticity matrices corresponding to two different, given solid elastic materials $* 1$ and $* 2$. Here, material $* 1$ is assumed to be the stiffer material, and material $* 2$ the softer one. It follows from (5) that for a given element, $\rho_{e}=1$ implies that the element fully consists of the solid material $* 1$, while $\rho_{e}=0$ means that the element fully consists of the solid material $* 2$. The symbol $r$ is the penalization power to penalize the intermediate values of the material volume density, and normally assigned the values 3 or 4 .
The element mass matrix of the bi-material model may be stated in a similar way as

$\mathbf{M}_{e}\left(\rho_{e}\right)=\rho_{e}^{q} \mathbf{M}_{e}^{* 1}+\left(1-\rho_{e}^{q}\right) \mathbf{M}_{e}^{* 2}$

where $\mathbf{M}_{e}^{* 1}$ and $\mathbf{M}_{e}^{* 2}$ are the element mass matrices corresponding to the two given solid materials as in (5). It is known that a pertinent value of the power $q$ is 1 in design for maximization of eigenfrequencies because penalization of the ratio between stiffness and mass (representing the squared eigenfrequency) is the important aspect in such a topology design problem (Pedersen 2000; Jensen and Pedersen 2006). In the present paper, the design objective of minimizing the dynamic compliance depends mainly on the dynamic stiffness $\mathbf{K}_{d}$ (defined as $\mathbf{K}_{d}=\mathbf{K}-\omega^{2} \mathbf{M}$ ) of the structure, especially in the case of high excitation frequencies. Thus, the same penalization is applied simultaneously to the stiffness and mass (i.e. to the dynamic stiffness $\mathbf{K}_{d}$ ) which leads to the following interpolation formula,

$\mathbf{K}_{d e}\left(\rho_{e}\right)=\rho_{e}^{r} \mathbf{K}_{d e}^{* 1}+\left(1-\rho_{e}^{r}\right) \mathbf{K}_{d e}^{* 2}$

Here, $\mathbf{K}_{d e}^{* 1}$ and $\mathbf{K}_{d e}^{* 2}$ are the elemental dynamic stiffness matrices corresponding to the two different, given solid elastic materials $* 1$ and $* 2$, and the penalization power $r$ was assigned the value 3 for both stiffness and mass in this paper, which resulted in distinctive optimum topology designs in the examples of bimaterial problems presented in Sections 5.1 and 5.2 of this paper. In these examples, the same density filtering technique (Sigmund 2007) as mentioned in Section 2.1.1, was applied in order to avoid checkerboard formation and dependency of topology solutions on finite element mesh-refinement.

Actually, the similar penalization model (i.e. penalizing the stiffness and the mass simultaneously) has been used in another joint work by the authors concerning vibro-acoustic design (see e.g. Du and Olhoff 2010). Besides this, introduction of the additional regularization term to the design objective (see. e.g. Allaire and Francfort 1993; Du and Olhoff 2010; Sigmund and Maute 2013) may also be helpful for improvement of obtaining a clear $0-1$ result in the dynamic design, and this is also implemented in our computer program.

Note that when bi-material design is treated via the problem formulation (1), then $V^{*}$ denotes the total volume $\sum_{e=1}^{N_{E}} \rho_{e} V_{e}$ of the stiffer material $* 1$ available for the structure, while the total volume of the softer material $* 2$ is given by $V_{0}$ $V^{*}$. Thus, in the topology optimization of bi-material structures, the entire admissible design domain will be filled-out by the two kinds of prescribed materials, and in figures presenting optimized topologies of bi-material structures, material $* 1$ is shown in black and material $* 2$ in grey. 
It should be noted that when the density filter is employed, the elemental volumetric material density $\rho_{e}$ in the formulations of Section 2 should be replaced by its filtered value $\tilde{\rho}_{e}=\frac{\sum_{i \in \bar{N}_{e}} w_{i} V_{i} \rho_{i}}{\sum_{i \in \bar{N}_{e}} V_{i} \rho_{i}}$. Here $V_{i}$ is the volume of the element $i$ and $\bar{N}_{e}$ is the neighborhoods of the element $e$ defined by $\bar{N}_{e}=$ $\left\{i \mid\left\|\mathbf{x}_{i}-\mathbf{x}_{e}\right\| \leq R\right\}$, where $\left\|\mathbf{x}_{i}-\mathbf{x}_{e}\right\|$ represents the distance between the centers of the element $i$ and element $e$, and $R$ is the filter radius. The weighting coefficient $w_{i}$ is defined by $w_{i}=R-\left\|\mathbf{x}_{i}-\mathbf{x}_{e}\right\|$. Correspondingly, the material density in the volume constraint 1 (d) should also be replaced by the filtered density to guarantee the volume preservation of the design (Bruns and Tortorelli 2001; Sigmund 2007).

\subsection{Localized vibration modes}

Application of the SIMP model for problems of singlematerial topology optimization with respect to dynamic structural response for prescribed excitation frequencies may lead to the occurrence of spurious, localized vibration modes. The reader is referred to slightly different methods of elimination of such spurious modes in vibrating, single-material structures in the papers Pedersen (2000), Tcherniak (2002) or Du and Olhoff (2007b). On the other hand, the problem of localized vibration modes very seldom manifests itself in bi-material design problems, since the localized vibration modes would be due to large differences in the specific stiffnesses in the different subdomains of the structure. In comparison with the single-material model, in the bi-material model the softer material $* 2$ normally has sufficient specific stiffness relative to the stiffer material $* 1$ to prevent occurrence of localized vibration modes. For example, in the numerical examples in Sections 5.1 and 5.2, the two prescribed materials employed have the same specific stiffness, i.e. $\frac{E_{m}^{* 2}}{\gamma_{m}^{2}}=\frac{E^{* 1}}{\gamma_{m}^{*} \text {, }}$, where $E^{* 1}$ and $\gamma_{m}^{* 1}$ are the Young's modulus and specific mass of the stiffer material $* 1$, and $E^{* 2}$ and $\gamma_{m}^{* 2}$ are the Young's modulus and specific mass of the softer material $* 2$. Thus, the problem of localized vibration modes may be avoided in these examples.

\subsection{Sensitivity analysis}

The sensitivity of the objective function $F$ in problem (1a-e) with respect to the design variables $\rho_{e}$ is given by

$F^{\prime}=\left(C_{d}^{2}\right)^{\prime}=2\left(\mathbf{P}^{\mathbf{T}} \mathbf{U}\right)\left(\mathbf{P}^{\prime \mathbf{T}} \mathbf{U}+\mathbf{P}^{\mathbf{T}} \mathbf{U}^{\prime}\right)$

where prime denotes partial derivative with respect to $\rho_{e}$ (or $\tilde{\rho}_{e}$ when the filtered density is introduced). The sensitivity $\mathbf{P}^{\prime}$ of the load vector will be zero if $\mathbf{P}$ is designindependent, otherwise it can be handled using the methods described by Hammer and Olhoff $(1999,2000)$ and $\mathrm{Du}$ and Olhoff $(2004 \mathrm{a}, \mathrm{b})$. The sensitivity $\mathbf{U}^{\prime}$ of the displacement vector is given by

$$
\left(\mathbf{K}-\omega^{2} \mathbf{M}\right) \mathbf{U}^{\prime}=\mathbf{f}=\mathbf{P}^{\prime}-\left(\mathbf{K}^{\prime}-\omega^{2} \mathbf{M}^{\prime}\right) \mathbf{U}
$$

where the sensitivities of the global stiffness and mass matrices (or the global dynamic stiffness matrix) can be directly obtained from the SIMP material models. The vector $\mathbf{f}$ is known as the pseudo load and is defined by the term on the right-hand side of (9). Instead of solving (9), the adjoint method (see e.g. Tortorelli and Michaleris (1994)) may be applied to calculate the sensitivity of the objective function in a more efficient manner, which gives the following result

$$
F^{\prime}=2\left(\mathbf{P}^{\mathbf{T}} \mathbf{U}\right)\left[2 \mathbf{U}^{\mathbf{T}} \mathbf{P}^{\prime}-\mathbf{U}^{\mathbf{T}}\left(\mathbf{K}^{\prime}-\omega^{2} \mathbf{M}^{\prime}\right) \mathbf{U}\right]
$$

Accordingly, the optimality condition for problem (1) can be expressed in the following form by means of the method of Lagrange multipliers,

$2\left(\mathbf{P}^{\mathbf{T}} \mathbf{U}\right)\left[2 \mathbf{U}^{\mathbf{T}} \mathbf{P}^{\prime}-\mathbf{U}^{\mathbf{T}}\left(\mathbf{K}^{\prime}-\omega^{2} \mathbf{M}^{\prime}\right) \mathbf{U}\right]+\Lambda V_{e}=0$,

where $\Lambda$ is the Lagrange multiplier corresponding to the material volume constraint, and the side constraints for $\rho_{e}$ have been ignored. The optimization problem (1a-e) can be solved by using the well-known MMA method (Svanberg 1987) or an optimality criterion method, e.g. the fixed point method, as devised by Cheng and Olhoff (1982).

When filtered density $\tilde{\rho}_{e}$ is introduced, the final sensitivity results may be derived using the chain rule as $\frac{\partial F}{\partial \rho_{e}}=\sum_{i \in \bar{N}_{e}} \frac{\partial F}{\partial \tilde{\rho}_{i}} \frac{\partial \tilde{\rho}_{i}}{\partial \rho_{e}}$, where $\frac{\partial \tilde{\rho}_{i}}{\partial \rho_{e}}=\frac{w_{e} V_{e}}{\sum_{j \in \bar{N}_{i}} w_{j} V_{j}}$.

\subsection{Incremental frequency technique (IF technique)}

The IF technique is applicable for both problems with 'low' and 'high' excitation frequencies. The IF technique can not only be used to determine the dependence of the minimized dynamic compliance on the excitation frequency over a range of such frequencies, but in the present paper, the IF technique provides an important means to drive a resonance frequency $\Omega_{m}$ of any order $m$ up or down by incremental changes of the variable excitation frequency $\omega$. The 'incremental frequency technique' is defined as follows:

IF technique: For given $\mathbf{P}$ solve sequentially the design problem in (1a-e) for minimum dynamic compliance $C_{d}$ subject to increasing (or decreasing) the variable 
excitation frequency $\omega$ in small incremental steps from a given initial value $\omega=\omega_{\text {init }}$ to a prescribed, final value $\omega=\omega_{\text {final }}=\omega_{p}$ by using as input for each step the incremented value of $\omega$ together with the topology design and the displacements obtained in the preceding step.

Following a discussion of the IF technique in the subsequent Section 2.5, it will be exemplified in Section 3 that the IF technique enables us to perform topology design for minimum dynamic compliance in a more dynamic and flexible manner in comparison with the conventional method where the excitation frequency is normally fixed at its prescribed value during the design process. This is not only true for a prescribed 'low', but also for a prescribed 'high' excitation frequency $\omega_{p}$. In Section 4, we shall then generalize and extend the IF technique to a systematic method termed the 'generalized incremental frequency method' (the GIF method) that can be applied to problems of topology optimization (as well as sizing and shape optimization) for minimum dynamic compliance (or e.g., minimum sound emission) in a more global manner by taking into account the multiple disjointed design sub-spaces of the problem irrespective of the value of the prescribed excitation frequency $\omega_{p}$.

\subsection{Discussion of IF technique based topology design of single-material structures}

The initial design for the topology optimization problem for minimum dynamic compliance without damping defined in (1a-e), is normally chosen to have a uniform distribution of material with intermediate density over the admissible design domain. This initial design may have a fundamental resonance frequency $\Omega_{1}$ which is either lower or larger than a prescribed initial excitation frequency $\omega=\omega_{\text {init }}$, cf. the sketches of the dynamic compliance $C_{d}$ for the initial design D1 in Fig. 1a and b. Here, $\omega_{\text {init }}$ is a 'high' excitation frequency in Fig. 1a and a 'low' excitation frequency in Fig. $1 \mathrm{~b}$.

\subsubsection{Case of $\Omega_{1}<\omega_{\text {init }}$}

Referring to Fig. 1xa, in the case of $\Omega_{1}<\omega_{\text {init }}$, the decrease of the dynamic compliance $C_{d}$ at $\omega=\omega_{\text {init }}$ normally implies an increase of the static compliance (that corresponds to the same loading amplitude but zero frequency $\omega=0$ ), due to the decrease of the fundamental resonance frequency $\Omega_{1}$ to the value $\Omega_{2}$ of the intermediate design D2, see Fig. 1a. As a result, in particular for single-material design, the structure may become very weak in terms of large static compliance. In order to prevent this, one may introduce an upper bound constraint on the static compliance $C_{s 2}$.

Assuming that such an upper bound is not considered, we may study the limiting case of $\omega=0$ by using the 'incremental frequency technique' (IF technique)
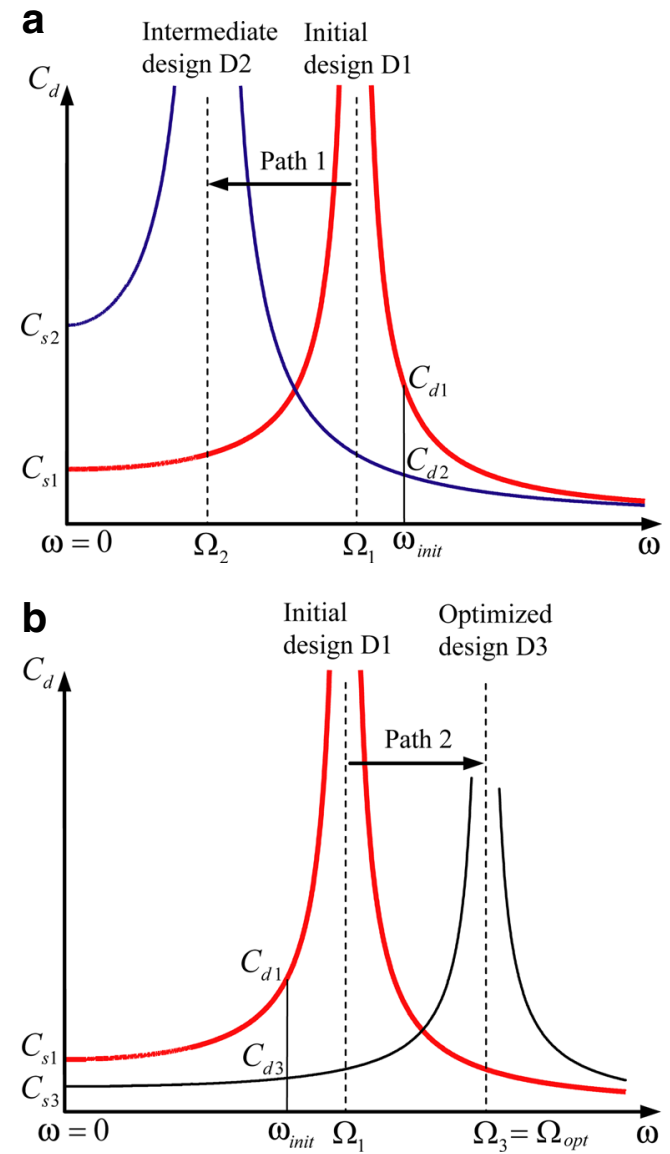

Fig. 1 Principle sketches indicating the dependence of the dynamic compliance $C_{d}$ on the excitation frequency $\omega$ for cases where the prescribed initial excitation frequency $\omega_{\text {init }}$ is high' in Fig. 1a and 'low' in Fig. 1b. relative to the fundamental resonance frequency $\Omega_{1}$ of the initial design D1. Symbols $\Omega_{2}$ and $\Omega_{3}$ represent the fundamental resonance frequencies of the designs D2 and D3, where the latter is associated with the maximum obtainable value for a 'low' excitation frequency. (a) If the fundamental resonance frequency $\Omega_{1}$ of the initial design D1 is less than the prescribed excitation frequency $\omega_{\text {init }}$, i.e., $\Omega_{1}<\omega_{\text {init }}$, then the design will proceed along path 1 (see Fig. 1a) to decrease the dynamic compliance. As a result, while the dynamic compliance corresponding to $\omega_{\text {init }}$ decreases, i.e. $C_{d 2}<C_{d 1}$, the static compliance $C_{s 2}$ (corresponding to $\omega=0$ ) increases, $C_{s 2}>C_{s 1}$, and the design 'degenerates' in the limit of $\omega=0$. (b) If $\Omega_{1}>\omega_{\text {init }}$, then the design will proceed along path 2 (see Fig. $1 \mathrm{~b}$ ), and as a result, both the dynamic compliance (corresponding to $\omega_{\text {init }}$ ) and the static compliance (corresponding to $\omega=0$ ) decrease, i.e. $C_{d 3}<C_{d 1}$ and $C_{s 3}<C_{s 1}$. Clearly, the latter case is preferable

defined in Section 2.4, to decrease the excitation frequency $\omega$ in small steps from an initial value $\omega=\omega_{\text {init }}$ that is slightly larger than the value $\omega=\Omega_{1}$ of the fundamental resonance frequency of the initial design D1, to a final value $\omega=\omega_{\text {final }}$ that is slightly larger than zero, cf. Fig. 1a. Please note that application of the IF technique includes solution of the minimization problem for the dynamic compliance $C_{d}$ in (1a-e) in each step, and that the input for a new step comprises the new, incremented value of $\omega$ together with the topology and 
displacements obtained in the preceding step. As indicated in Fig. 1a, the result is that the minimization of the dynamic compliance drives the fundamental resonance frequency of the design towards zero, and, at the same time, the static displacements of the structure become very large, which means that the static compliance tends to infinity. The physical interpretation of this behaviour is that in the limit of $\omega=0$, a disintegration is created in the structure. In this limit, the zero value of the fundamental resonance frequency is associated with a rigid body vibration mode of the structure, and the static displacements of the disintegrated part of the structure become infinite, as the structure cannot sustain the static load. A straight-forward way of avoiding this type of 'degenerate' structural design is to include an upper bound constraint on the static compliance in the mathematical formulation (1a-e) of the problem of minimizing the dynamic compliance. Our tests have shown that such a constraint is extremely effective and wellchosen for the case discussed above.

\subsubsection{Case of $\Omega_{1}>\omega_{\text {init }}$}

We now consider the case depicted in Fig. 1b, where the fundamental resonance frequency $\Omega_{1}$ of the initial design D1 is larger than a prescribed initial excitation frequency $\omega=\omega_{\text {init }}$. In this case we follow path 2 , and as indicated in Fig. 1b, not only the dynamic compliance $C_{d}$ (corresponding to $\omega=\omega_{\text {init }}$ ), but also the static compliance $C_{s}$ (corresponding to $\omega=0$ ) are decreased.

In this case, we may apply the IF technique (see Section 2.4) to increase the excitation frequency $\omega$ in small steps from an initial value $\omega_{\text {init }}$ that is smaller than the fundamental resonance frequency $\Omega_{1}$ of the initial design D1, to a final value $\omega=\omega_{\text {final }}$ that is slightly less than the shown optimized fundamental resonance frequency $\Omega_{3}=\Omega_{\mathrm{opt}}$ of the optimized design D3. Please recall that the fundamental resonance frequency $\Omega_{3}=\Omega_{\mathrm{opt}}$ and its associated optimized topological design can be computed as the solution to the physically slightly different problem of maximizing the fundamental (first) eigenfrequency of free vibrations ( $\mathrm{Du}$ and Olhoff 2007b, c).

The above IF technique has the desirable effect of generating a series of topologies with increasing values of both the fundamental resonance frequency and the static and dynamic stiffness for the sequence of structures produced. The technique automatically avoids resonance, and works very well as long as the prescribed final excitation frequency $\omega_{\text {final }}$ is lower than the maximum obtainable value $\Omega_{\text {opt }}$ of the fundamental resonance frequency, i.e. $\omega_{\text {final }}<\Omega_{\text {opt }}$. Moreover, it can be easily proved that the dynamic stiffness matrix $\mathbf{K}_{d}=\mathbf{K}-\omega^{2} \mathbf{M}$, see (1c), will remain positive definite if the fundamental resonance frequency (equaling the fundamental eigenfrequency of free vibrations) of the structure maintains a value that is higher than the excitation frequency during the design process (i.e. Case of Fig. 1b). This implies a very good feature embedded in the dynamics design, i.e., the structural response will approach zero if the dynamic compliance of the structure approaches zero. In addition, according to our experiences, the iterative numerical process in the dynamics design will be more stable for a positive definite dynamic stiffness matrix $\mathbf{K}_{d}$.

\section{Numerical examples - minimum dynamic compliance design of a plate-like structure}

This section presents two numerical sub-examples of design of the plate-like, single-material structure shown in Fig. 2a. A main objective is to investigate two different design paths for the optimization and to achieve a physical interpretation of the results.

A time-harmonic, concentrated transverse external load $p(t)=P \cos \omega t$ is applied to the center of the plate. The design objective is to minimize the dynamic compliance of the plate for a prescribed 'low' external excitation frequency $\omega=\omega_{p}=80$ and a volume fraction of $50 \%$ for the given solid material, which has Young's modulus $E=10^{11}$, Poisson's ratio $v=0.3$ and the specific mass $\gamma_{m}=7800$. A time-harmonic, concentrated transverse external load $p(t)=P \cos \omega t$ is applied to the center of the plate. The design objective is to minimize the dynamic compliance of the plate for a prescribed excitation frequency $\omega=\omega_{p}=80$ and a volume fraction of $50 \%$ for the given solid material, which has Young's modulus $E=10^{11}$, Poisson's ratio $v=0.3$ and the specific mass $\gamma_{m}=7800$. SI units are used throughout this paper.

The finite element model of the plate-like structure consists of $600(30 \times 20 \times 1) 8$-node $3 \mathrm{D}$ brick elements with Wilson incompatible displacement models (Wilson et al. 1973) to improve precision.

\subsection{Sub-example 1 - 'degenerate' design}

With reference to Fig. 1a and Section 2.5.1, the fundamental resonance frequency of the plate in its initial design (see Fig. 2a) is found to be $\Omega_{1}=61.6$, i.e., less than the prescribed excitation frequency $\omega_{p}=80$. Adopting the IF technique (Section 2.4) and the design path 1, we find that the minimization of the dynamic compliance leads to increasing distance between $\omega_{p}$ and $\Omega_{1}$ as shown in the iteration history in Fig. 2b. At the same time, the dynamic compliance tends to zero, and the static compliance of the structure increases very quickly (Fig. 2c). Figure $2 \mathrm{~d}$ shows that at iteration step 30 , the plate has become very 


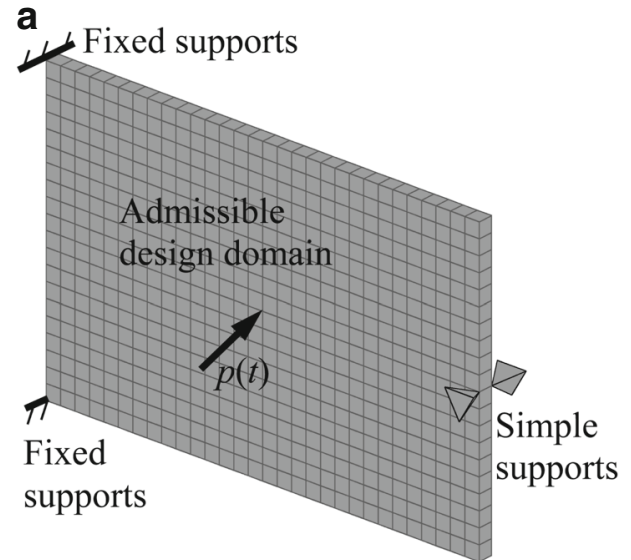

b

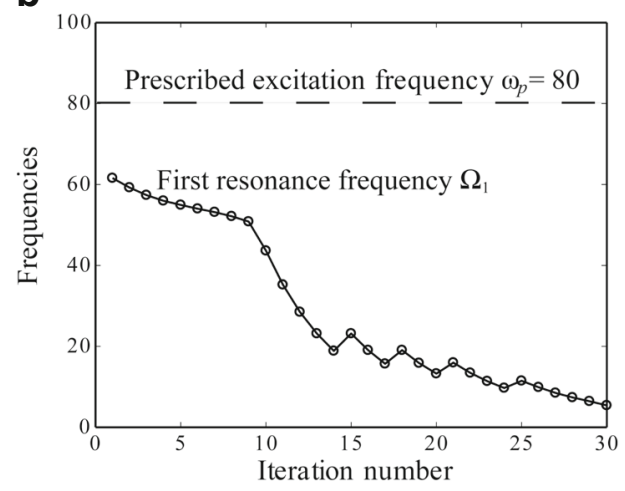

Fig. 2 (a) Admissible design domain $(a=3, b=2$ and $c=0.1)$ with loading and support conditions. (b) Iteration history for the first resonance frequency $\Omega_{1}$ of the plate $\left(\Omega_{1}<\omega_{p}=80\right)$. (c) Iteration histories for the dynamic and static structural compliance (the latter

weak at the two fixed supports. This indicates creation of a rigid body vibration mode in association with vanishing of the fundamental resonance frequency, and that the structure cannot effectively sustain the static load associated with $\omega=0$.

A straight-forward way of avoiding this kind of 'degeneracy' of the structure is to include an upper bound constraint on the static compliance in the mathematical formulation (1a-e) of the problem of minimizing the dynamic compliance. This has an increasing effect on the dynamic compliance (and computational time), but our performed numerical tests have shown that such a constraint is otherwise very effective and well-chosen for the purpose.

\subsection{Sub-example 2 - desirable design}

Referring to Fig. $1 \mathrm{~b}$ and Section 2.5.2, we may adopt the much more expedient design path 2 that avoids the 'degeneracy' of the design. Thus, let us again apply the IF technique, but now start out the design problem with a value $\omega=\omega_{\text {init }}$ of the excitation frequency that is less
C

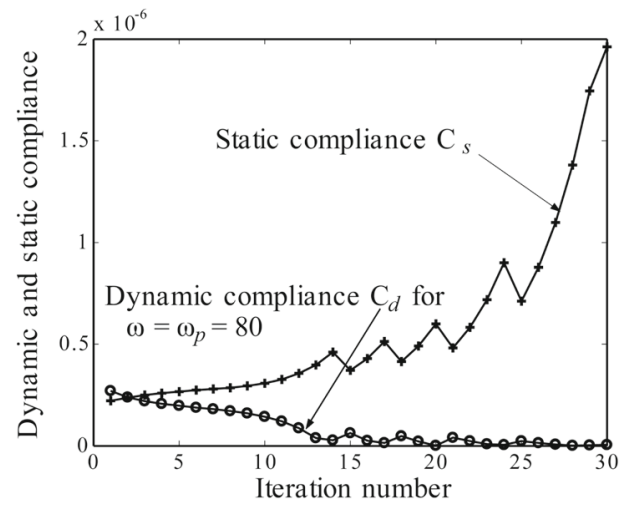

d

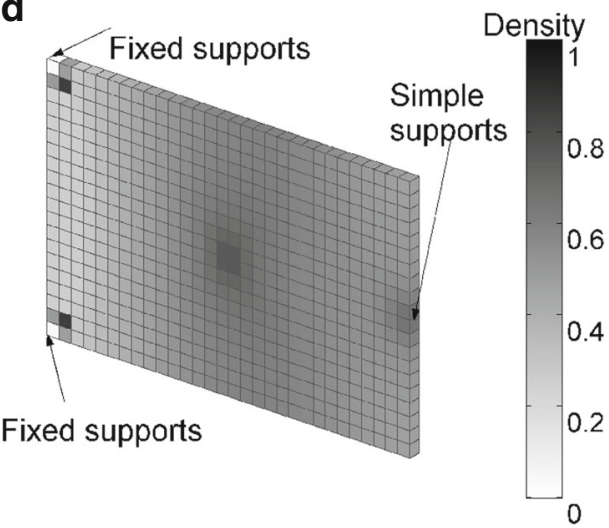

corresponds to the same loading amplitude, but excitation frequency $\omega=0$ ). (d) Material distribution with very weak sub-domains near the two fixed supports at iteration step 30

than the first eigenfrequency $\Omega_{1}$ of the initial design, and then sequentially increase $\omega$ up to its originally prescribed value $\omega=\omega_{p}=80$ (Fig. 3a). The converged design, which is shown in Fig. 3c, is a much more desirable structure with minimized dynamic compliance and improved static stiffness, see Fig. 3a, b.

It is worth noting a special case that may occur if the prescribed value of the excitation frequency is just slightly lower than the maximum fundamental resonance frequency. Then the IF technique might fail to reach the prescribed value of the excitation from the left-hand side of the maximum fundamental resonance frequency (i.e. the design performed along path 2 , cf. e.g. Fig. 1b). If this happens, we have to perform the design along another path 1 (cf. e.g. Fig. 1a). For single material designs, the design path 1 , as discussed earlier, may lead to the 'degenerated design' that is statically too weak to be applied in engineering practice. Thus, for this special case, it is suggested to use the common and straightforward way, i.e. to introduce an additional proper upper bound constraint on the static compliance in order to avoid the 'degeneracy' of the design. Actually, such an additional constraint is also useful for single-material design 
a

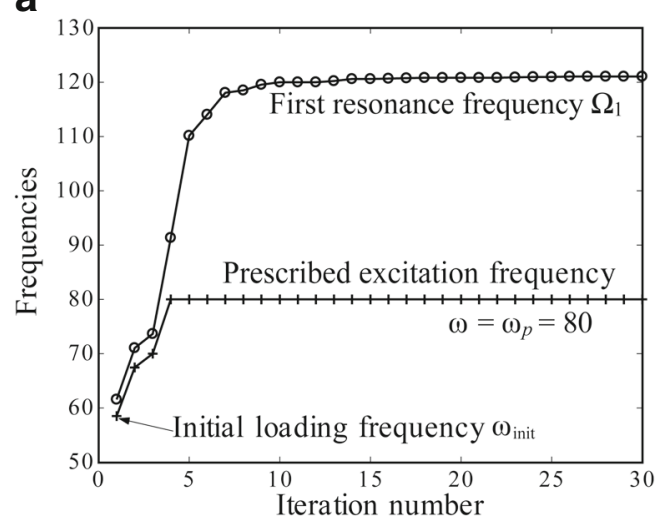

b

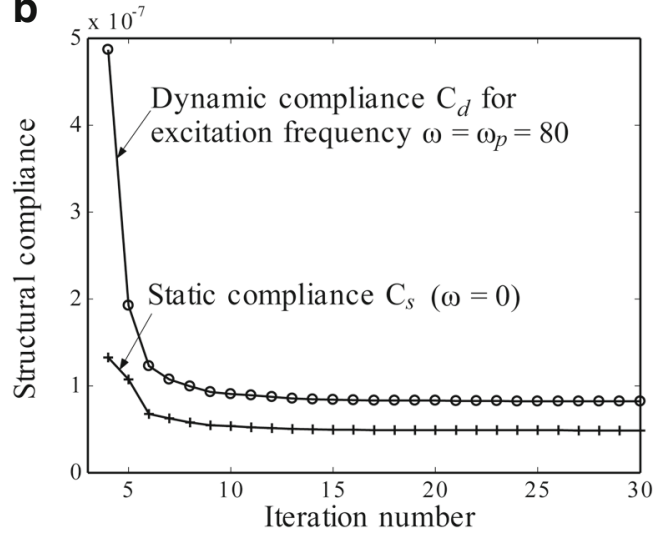

C
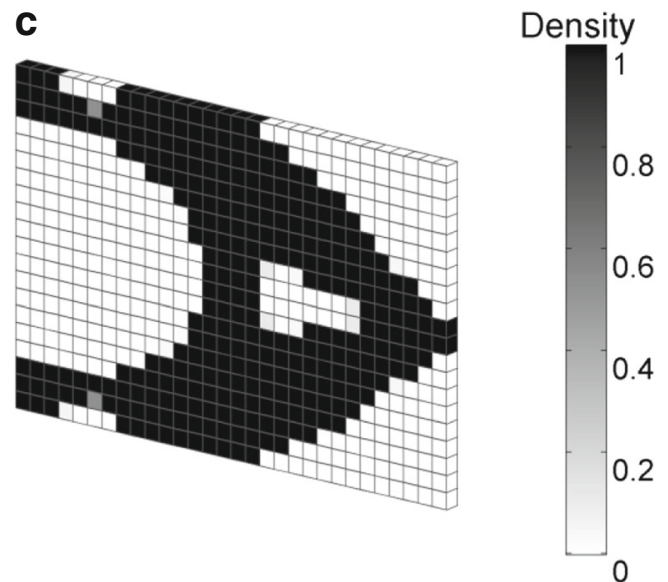

Fig. 3 (a), (b) Iteration histories for the first resonance frequency of the plate, the excitation frequency, and the dynamic and static compliances. (c) Optimized topology (50\% volume fraction) for $\omega_{p}=80$

subjected to a high excitation frequency as mentioned in the last part of Section 4.1.1.

\section{Development of generalized incremental frequency method (GIF method)}

Let us first present an outline of the background for the GIF method with reference to Fig. 4 that depicts the dependence of

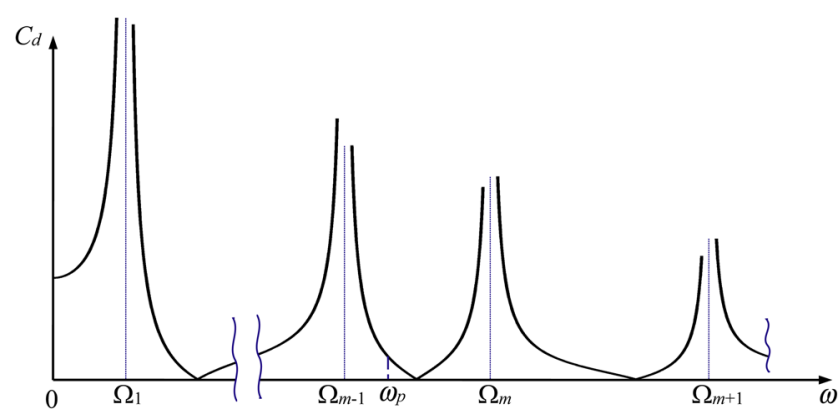

Fig. 4 Principle sketch of the dependence of the dynamic compliance $C_{d}=\left|\mathbf{P}^{\mathrm{T}} \mathbf{U}\right|$ on the loading frequency $\omega$ for a given initial topological design and a given vector $\mathbf{P}$ of amplitudes of the external forces. The resonance frequencies $\Omega_{1}, \Omega_{m-1}, \Omega_{m}$ and $\Omega_{m+1}$ of the design are shown together with a prescribed, 'high' external excitation frequency $\omega_{p}$

the dynamic compliance $C_{d}=\left|\mathbf{P}^{\mathrm{T}} \mathbf{U}\right|$ of a given design on the loading frequency $\omega$ of an external time-harmonic mechanical surface loading with the given vector $\mathbf{P}$ of amplitudes acting on the surface, resulting in a vector $\mathbf{U}$ of amplitudes of the displacement response.

In Fig. $4, \Omega_{i}, i=1, \ldots, m-1, m, m+1$, denote resonance frequencies of the given design, and $\omega_{p}$ is a prescribed external excitation frequency that is indicated to be 'high' in the figure. Regarding the resonance frequencies, it is important to remind that while a resonance frequency of a forced time-harmonic vibration problem is at the same time an eigenfrequency of the corresponding problem of free vibrations (and can be computed as such), an eigenfrequency may not necessarily be a resonance frequency. Thus, if the eigenvector $\varphi_{i}$ associated with an eigenfrequency is orthogonal to the vector of load amplitudes $\mathbf{P}$ of the forced vibration problem, i.e., if

$\mathbf{P}^{\mathbf{T}} \boldsymbol{\varphi}_{i}=0$

then the eigenmode cannot be excited. In such cases, the eigenfrequency will not constitute a resonance frequency of the forced vibration problem, and it will not affect the behaviour of the dynamic compliance $C_{d}$, cf. Fig. 4 .

As illustrated in Fig. 4, which is depicted for a given topological design, the dynamic compliance $C_{d}=\left|\mathbf{P}^{\mathbf{T}} \mathbf{U}\right|$ is a nonnegative function of the excitation frequency $\omega$. Thus, $C_{d}$ vanishes at each of the anti-resonance points between the adjacent resonance frequencies located at the left- and righthand sides of the anti-resonance point, and $C_{d}$ increases uniformly towards infinity at the resonance frequencies as material damping is not considered in this study. These characteristics imply that a solution to the 'conventional' dynamic compliance minimization problem (1a-e) subject to a fixed, prescribed excitation frequency $\omega_{p}$ will furnish a local optimized solution where the given excitation frequency $\omega_{p}$ always will be located between two consecutive resonance frequencies that have different values, but the same orders as those of the initial design chosen for the computational procedure. 
However, as indicated in Fig. 4, the current frequency response problem is non-convex and complicated by having disjointed design sub-spaces (i.e. sub-intervals) between the resonance frequencies $\Omega_{i}, i=1, \ldots, m-1, m, m+1, \ldots$, and we must consider the possibility that there may very well exist one or more 'better' local optimized designs (associated with lower values of $C_{d}$ ), for which the prescribed excitation frequency $\omega_{p}$ is located between a pair of consecutive resonance frequencies of other orders than those of the aforementioned local optimized design obtained by just solving (1a-e) subject to the given value $\omega_{p}$. Not surprisingly, it turns out that by simple application of the IF technique defined in Section 2.4, one may obtain a number of different local optimized topology designs with a given excitation frequency $\omega_{p}$ located in sub-intervals between pairs of consecutive resonance frequencies of different orders than those of the initial design.

As there are limits to how much resonance frequencies (like eigenfrequencies of the corresponding free vibration problem) can be increased or decreased due to the constraints considered (see Du and Olhoff 2007b), the number of the different local optimized topology designs of the forced vibration problem will generally be rather small. Thus, by simply identifying the topology design associated with the smallest value of $C_{d}$ from among this small number of local optimized designs, we can determine the design that may be considered the global optimum solution to the problem.

Finally, it is important to mention that the non-convexity of the design problem normally implies that the optimized topology exhibits dependence on the choice of the initial design. A conventional way of searching for the global solution for such kind of problem is to start the design with some different (e.g. randomly generated) initial designs. However, for the dynamic problem considered in the present paper, if the excitation frequency is fixed at its prescribed value, our numerical tests have shown that, although the optimized topologies obtained from randomly generated different initial designs are different from each other in some details, they normally show some kind of similarity in topological concept relative to that obtained by using a uniform initial design. The more serious problem of the conventional design method is that the results obtained using the fixed excitation frequency cannot reflect well in a systematic manner the effects of different resonance frequency intervals of the frequency response curve, which the authors believe should play a more important role in searching for the global solution of the dynamic design problem and should be addressed carefully.

\subsection{Computational procedure of the GIF method}

The remainder of this paper will continue the preceding lines of analyses and disregard the abovementioned dependency of the design results on randomly generated initial designs, and thereby yield consistent results.
A computational procedure for the GIF method for determining the optimum topological design associated with minimum dynamic compliance subject to any prescribed positive value of the excitation frequency $\omega_{p}$ may be stated as below. The procedure is a single, unified algorithm for handling of both 'low' $(m=1)$ and 'high' $(m>1)$ excitation frequencies $\omega_{p}$ by the GIF method, cf. Fig. 4.

Step 1 Choose a convenient initial design with, e.g., a uniform distribution of the given amount(s) of material(s) over the admissible design domain, and subjected to the given boundary conditions.

Step 2 Compute the eigenfrequencies of free vibrations of the initial design from zero and up to a value well above the prescribed value $\omega_{p}$ of the excitation frequency of the forced vibration problem.

Step 3 Discard those eigenfrequencies of the initial design whose eigenvectors $\varphi_{i}$ are orthogonal (cf. (12)) to the amplitude vector function $\mathbf{P}$ of the external dynamic loading.

Step 4 Denote the remaining eigenfrequencies as resonance frequencies. Count these without possible multiplicities, and order them according to magnitude as

$\Omega_{0}=0<\Omega_{1}<\Omega_{2}<\cdots$

where, for convenience, $\Omega_{0}$ denotes zero. Assume that none of the positive resonance frequencies of the initial design are strictly equal to the prescribed value $\omega_{p}$ of the excitation frequency, cf. Fig. 4. Denote by $\Omega_{m}$ (with $m>1$ ) the nearest resonance frequency of the initial design that is larger than $\omega_{p}$. Referring to Fig. 4, please bear in mind that for the initial design the excitation frequency $\omega$ is defined to be 'low' for positive values up to and including the fundamental (first) resonance frequency of the design, and to be 'high' for values of $\omega$ beyond that.

Step 5 For the initial design chosen, and the given amplitude and spatial distribution of the external dynamic loading, solve iteratively the design problem (1a-e) for minimum dynamic compliance $C_{d}$ of the initial design subject to keeping the prescribed value $\omega_{p}$, $\Omega_{m-1}<\omega_{p}<\Omega_{m}$, of the excitation frequency fixed during the iterations.

Set the counter $N$ of local optimum solutions to $N=1$, save the topological design and the corresponding minimized value of the dynamic compliance $C_{d}$, and continue.

Step 6 If $m=1$ (i.e., if $\omega_{p}$ is smaller than the fundamental resonance frequency $\Omega_{1}$, cf. Fig. 4), then go to Step 8 .

Step 7 Starting with $i=\mathrm{m}-1$, apply the IF technique (see Section 2.4) to the initial design with an initial value $\omega_{\text {init }}$ of the excitation frequency $\omega$ that is slightly 
smaller than the resonance frequency $\Omega_{i}$ of the initial design, and increase $\omega$ in small steps towards its originally prescribed value $\omega=\omega_{\text {final }}=\omega_{p}$.

Set $Y=0$. If it is possible to increase $\omega$ to the value $\omega=\omega_{p}$ by the IF technique, then set $\mathrm{Y}=1$ and the counter of local optimum solutions $N=N+1$, save the topological design and the corresponding minimized value of the dynamic compliance $C_{d}$ obtained at $\omega_{p}$.

If $\mathrm{Y}=1$ and $i>1$ then repeat Step 7 with $i=i-1$ until $\mathrm{Y}=0$ has been obtained.

Step 8 Set $i=m$ and continue.

Step 9 Starting with $i=\mathrm{m}$, apply the IF technique (see Section 2.4) to the initial design with an initial value $\omega_{\text {init }}$ of the excitation frequency $\omega$ that is slightly larger than the resonance frequency $\Omega_{i}$ of the initial design, and decrease $\omega$ in small steps towards its originally prescribed value $\omega=\omega_{\text {final }}=\omega_{p}$.

Set $Y=0$. If it is possible to decrease $\omega$ to the value $\omega=\omega_{p}$ by the IF technique, then set $\mathrm{Y}=1$ and the counter of local optimum solutions $N=N+1$, save the topological design and the corresponding minimized value of the dynamic compliance $C_{d}$ obtained at $\omega_{p}$.

If $\mathrm{Y}=1$ then repeat Step 9 with $i=i+1$ until $\mathrm{Y}=0$ has been obtained.

Step 10 From among the total number $N$ of different local optimized topology designs obtained in Steps 5, 7 and 9 (only in Steps 5 and 9 if $m=1$ ), we finally identify the topology design that is associated with the smallest value of $C_{d}$, which we may consider as the global optimized topological design for the problem.

\subsubsection{Comments on the computational procedure of the GIF method}

The following comments should be made regarding the computational procedure presented above for the GIF method.

It is interesting to note that the algorithm for the GIF method in Section 4.1 is directly applicable for problems with prescribed 'high' excitation frequencies $\omega_{p}(m>1)$, and that the case of 'low' excitation frequencies $\omega_{p}(m=1)$ is very simply integrated in the procedure. The only difference is that Step 7 shall be skipped if $m=1$.

In Steps 2-4 an appropriate number of resonance frequencies of forced vibration of the initial design is determined by computing eigenfrequencies of free vibration of this design, and discarding those with eigenmodes $\mathbf{U}$ that are orthogonal to the given load amplitudes $\mathbf{P}$ of the forced vibration problem. This procedure is usually preferable from the point of view of computation time. An alternative approach is to generate, in similarity with Fig. 4, a pointwise representation of the dynamic compliance $C_{d}=\left|\mathbf{P}^{\mathrm{T}} \mathbf{U}\right|$ of the initial design by increasing the excitation frequency $\omega$ in small steps within a sufficiently large interval of interest for treatment of a problem with a prescribed 'high' value of the excitation frequency $\omega_{p}$. Generally, however, this is more costly because the steps in $\omega$ need to be chosen quite small, in particular in the vicinities of the resonance frequencies, such that these frequencies can be captured and be determined with reasonable accuracy.

In Step 5 the 'conventional' optimization problem (1a-e) is simply solved for the initial design subject to the fixed, prescribed value of $\omega=\omega_{p}$, i.e., no IF technique is performed for $\omega$, and the prescribed excitation frequency $\omega_{p}$ will be located between $\Omega_{m-1}$ and $\Omega_{m}$ if $m>1$, and between $\Omega_{0}$ and $\Omega_{1}$ if $m=1$.

The aim is now to sequentially identify intervals between consecutive resonance frequencies of the initial design that in the nearest lower and upper neighbourhoods of the interval $\left[\Omega_{m-1}, \Omega_{m}\right]$ embracing $\omega_{p}$, can contribute locally optimized designs and the corresponding minimized values of $C_{d}$ by optimizing the positions (values) of the resonance frequencies.

From Step 6, we skip Step 7 if $m=1$, but continue with Step 7 if $m>1$. Step 7 , which is started with the initial value $i=m-$ 1 , investigates sequentially for $i=m-1, m-2$, etc., whether it is possible by usage of the IF technique (Section 2.4) to 'push', one by one, the nearest resonance frequencies $\Omega_{i}$, $i=m-1, m-2$, etc., up to the prescribed value of the excitation frequency $\omega_{p}$. The resonance frequencies $\Omega_{i}$, for which this may be possible, will be the end points for a generally quite small number of neighbouring intervals $\left[\Omega_{m-2}, \Omega_{m-1}\right]$, $\left[\Omega_{m-3}, \Omega_{m-2}\right], \ldots$ below $\omega_{p}$. Each of such two frequency intervals will be the locus of a local optimized solution that furnishes the local optimized topological design and the corresponding minimized value of the dynamic compliances $C_{d}$ associated with the prescribed value of $\omega_{p}$. Notice that if, e.g., the resonance frequency $\Omega_{m-2}$ cannot be 'pushed' up to the value of $\omega_{p}$, then the local optimization has failed in the frequency interval $\left[\Omega_{m-3}, \Omega_{m-2}\right]$, and the design and value of $C_{d}$ obtained will not be considered among the local candidates for the global optimum solution.

The next Steps 8 and 9 are valid for both $m=1$ and $m>1$, and Step 9 is quite analogous to Step 7. Thus, Step 8 delivers the initial value $i=m$ for sequential investigations with $i=m$, $m+1, m+2$, etc. in Step 9, with a view to find out whether it is possible by usage of the IF technique (Section 2.4) to 'push' sequentially the nearest resonance frequencies, e.g., $\Omega_{i}, i=m$, $m+1$, etc. down to the prescribed value of the excitation frequency $\omega_{p}$. If this is possible, then the two resonance frequency intervals $\left[\Omega_{m}, \Omega_{\mathrm{m}+1}\right]$ and $\left[\Omega_{\mathrm{m}+1}, \Omega_{m+2}\right]$ will be the loci of two local optimized solutions, each with a local optimized topological design, the corresponding minimized value of the dynamic compliances $C_{d}$, and the value of $\omega_{p}$ considered. On the other hand, if , e.g., the resonance frequency $\Omega_{m+1}$ 
cannot be 'pushed' down to the value of $\omega_{p}$, then the frequency interval $\left[\Omega_{\mathrm{m}+1}, \Omega_{m+2}\right]$ will not be the locus of a local optimized solution.

In Step 10 we collect the different local optimized topology designs and corresponding minimized values of $C_{d}$ associated with the prescribed excitation frequency $\omega_{p}$. It is our experience that in general this number of local candidate designs will be rather small. Thus, by simply identifying the topology design associated with the smallest dynamic compliance from among the different local optimized solutions corresponding to $\omega_{p}$, it is straight-forward to select the topological design that may be considered as the 'global' optimized solution to the problem.

Finally, it is worth noting that for problems of topology optimization of bi-material structures (that are dealt with in the numerical examples in Section 5), the extended SIMP model in Section 2.1.2 implies that the entire design domain will be fully covered by the stiffer and the softer material, please refer to the last paragraph of Section 2.1.2. Thus, a disintegration of the structure and formation of a rigid-body mode associated with the fundamental frequency will not occur, so the static compliance will always be finite, and it will not be imperative to consider an upper bound constraint on the static compliance when minimizing the dynamic compliance of a bi-material structure. This type of problem is directly solvable by the algorithm in Section 4.1 for both $m=1$ and $m>1$.

For problems of topology optimization of single-material structures, of which numerical examples are presented in Section 3, a proper upper bound constraint on the static compliance may be included in the mathematical formulation (1a-e) of the problem of minimizing the dynamic compliance with a view to avoid possible static 'degeneracy' of the design.

Since a static compliance constraint may be very useful for both single- and bi-material structures, we introduce into (1ae) the additional equations

$C_{s}=\mathbf{P}^{\mathrm{T}} \mathbf{U}^{*} \leq \bar{C}_{s}$

$\mathbf{K} \mathbf{U}^{*}=\mathbf{P}$

Here, $\left(1 b^{*}\right)$ expresses the constraint for the static compli-

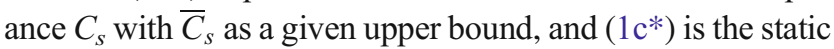
equilibrium equation, where $\mathbf{P}$ denotes the static loading, and the corresponding static displacement vector $\mathbf{U}^{*}$ is defined as $\mathbf{U}^{*}=\mathbf{U}(\omega=0)$. Based on $\left(1 \mathrm{~b}^{*}\right)$ and $\left(1 \mathrm{c}^{*}\right)$, the sensitivity $C_{S}^{\prime}$ of the static compliance is obtained as

$C_{s}^{\prime}=\mathbf{P}^{\prime \mathbf{T}} \mathbf{U}^{*}+\mathbf{P}^{\mathrm{T}} \mathbf{U}^{\prime *}=2 \mathbf{U}^{* \mathrm{~T}} \mathbf{P}^{\prime}-\mathbf{U}^{* \mathrm{~T}} \mathbf{K}^{\prime} \mathbf{U}^{*}$

where prime denotes partial derivative with respect to the design variable $\rho_{e}$, and the sensitivity $\mathbf{P}^{\prime}$ of the load vector vanishes if $\mathbf{P}$ is design-independent. Note that the gradient $C_{s}^{\prime}$ in (14) is reduced analogously to the gradient of the objective function $F$, for the squared dynamic compliance in (10) to facilitate treatment by adjoint sensitivity analysis.

With the inclusion of the upper bound on the static compliance of a structure, the dynamic and static equilibrium (1c) and $\left(1 \mathrm{c}^{*}\right)$ are solved by Gauss elimination, and the constrained topology design problem $\left(1 \mathrm{a}-\mathrm{e}, \mathrm{b}^{*}, \mathrm{c}^{*}\right)$ is solved iteratively to convergence by means of the gradients

$C_{d}^{\prime}$ and $C_{s}^{\prime}$ of the dynamic and static compliances, by usage of the MMA optimizer (Svanberg 1987).

To develop a single unified procedure that addresses the low and the high frequency cases along with single- and bimaterial structures for problems of minimizing the dynamic compliance subject to an upper bound constraint on the static compliance, we first change slightly the definition of the IF technique in Section 2.4 to the following definition of a socalled 'constrained IF technique':

'Constrained IF technique' with an upper bound specified for the static compliance: For given $\mathbf{P}$ solve sequentially the constrained design problem in (1a$\left.\mathrm{e}, \mathrm{b}^{*}, \mathrm{c}^{*}\right)$ for minimum dynamic compliance $C_{d}$ subject to increasing (or decreasing) the variable excitation frequency $\omega$ in small incremental steps from a given initial value $\omega=\omega_{\text {init }}$ to a prescribed, final value $\omega=\omega_{\text {final }}=\omega_{p}$ by using as input for each step the incremented value of $\omega$ together with the topology design and the displacements obtained in the preceding step.

Now, if we in the Steps 5, 7 and 9 of the algorithm of the GIF method in Section 4.1 replace the text strings 'design problem (1a-e)' by 'constrained design problem (1a-e, $\mathrm{b}^{*}, \mathrm{c}^{*}$ )' and 'IF technique (see Section 2.4)' by 'constrained IF technique (see above)', then the algorithm in Section 4.1 is easily transferred to the desired single, unified algorithm aimed at. For reasons of brevity, we have omitted to write the algorithm in full here.

Please note that with the extension of (1a-e) by $\left(1 b^{*}, c^{*}\right)$, use of the constrained IF technique and replacement of the above text strings, then the static compliance $C_{S}$ appears very clearly in the new, extended algorithm and makes it very simple to avoid occurrence of 'degenerate' designs.

\subsubsection{Flow chart of the GIF method}

For the convenience of programming, a flow chart of the GIF method is designed and shown in Fig. 5. The flow chart consists of three cycles, i.e. the inner MMA cycle, the inner 'Constrained IF technique' cycle, and the global cycle of the GIF method. From consideration of computational efficiency, the MMA cycle will be performed only one time in each cycle 


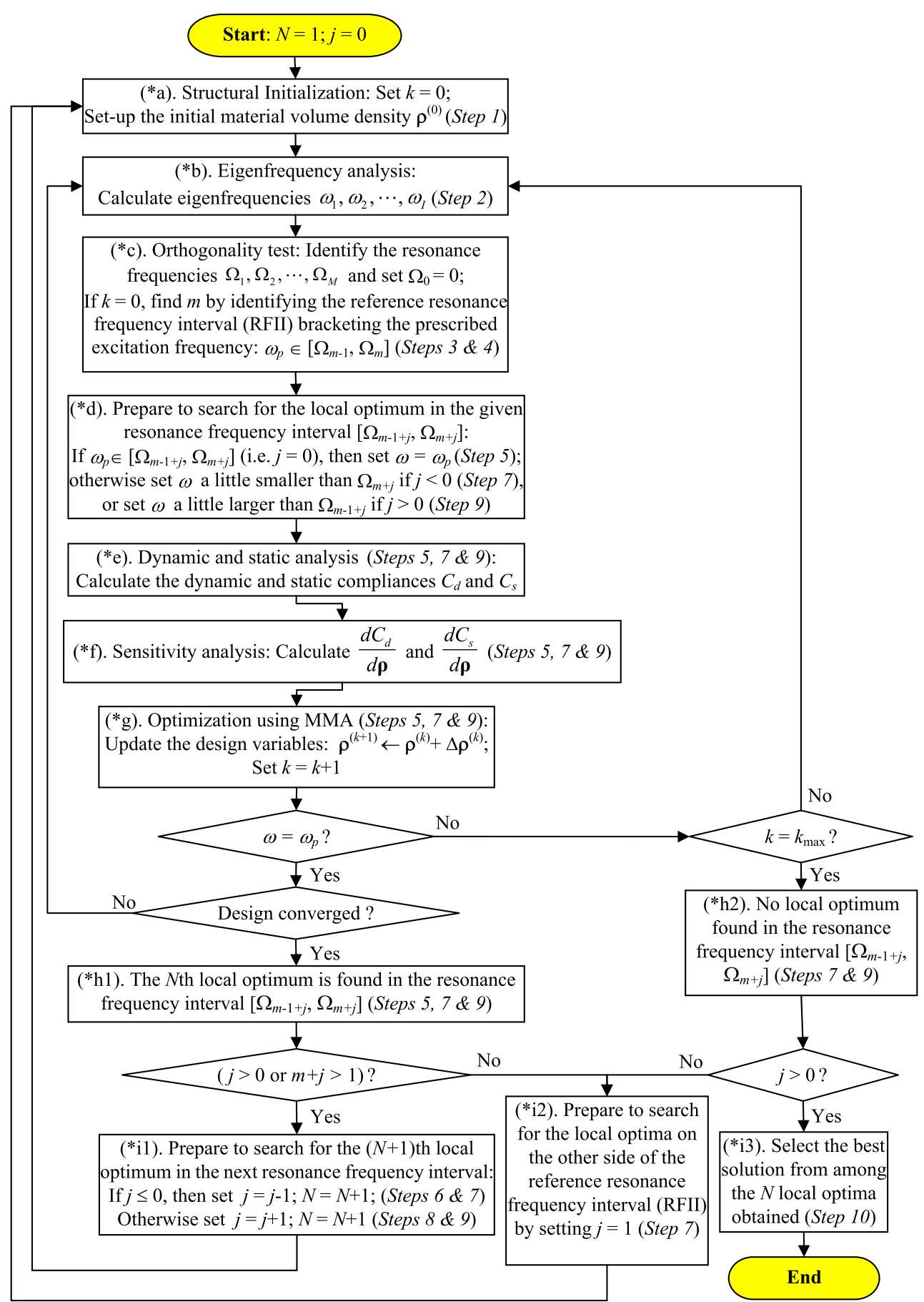

Fig. 5 Flow chart of the 'generalized incremental frequency method' (GIF method)

of the 'Constrained IF technique' before the excitation frequency $\omega$ reaches the prescribed value $\omega_{p}$.

In the flow chart, the symbol $N$ is a counter of the number of obtained local optimized designs, and $k$ counts the number of the IF technique cycles. Moreover, the index $m$ represents the order of the upper resonance frequency $\Omega_{m}$ of the resonance frequency interval of the initial design (RFII), that brackets the prescribed, external excitation frequency $\omega_{p}$, cf. Fig. 5.
In accordance with the computational procedure of the GIF method outlined in Section 4.1, in the present flow chart, the order of searching for local optima is designated as starting search (with $j=0$ ) from the resonance frequency interval of the initial design (RFII), and then searching for local optima in the sequential resonance frequency intervals (with decreasing negative integer values $-1,-2, \ldots$ of $j$ ) at the left-hand side of the RFII. This searching is stopped the first time when no solution is found in one of the sequential resonance frequency 
intervals at the left-hand side of the RFII, or the searching in the interval $\left[\Omega_{0}, \Omega_{1}\right]$ is completed.

Next, searching for local optima in the sequential resonance frequency intervals (with increasing positive integer values $1,2, \ldots$ of $j$ ) at the right-hand side of the RFII is carried out, and the global cycles of the GIF method will be terminated the first time when no solution is found in one of the sequential resonance frequency intervals at the right-hand side of the RFII.

In order to fit the notion of flow chart, the computational procedure of the GIF method in Section 4.1 is restated using 12 sub-steps denoted from $(* a)$ to $(* i 3)$ as shown in Fig. 5. Here, the sub-steps $(* \mathrm{a}),(* \mathrm{~b})$ and $(* \mathrm{c})$ correspond to Steps 1,2 and 3 of Section 4.1 by performing initialization of the structure and identification of the resonance frequencies and the resonance frequency interval of the initial design (RFII) bracketing the prescribed excitation frequency $\omega_{p}$. Sub-step $(* d)$ sets the initial value of the excitation frequency $\omega$ for the IF technique cycle according to the current resonance frequency interval selected for searching for the local optimum, which corresponds to the first parts of Steps 5, 7 and 9 of Section 4.1. Sub-steps $(* \mathrm{e}),(* \mathrm{f}),\left({ }^{*} \mathrm{~g}\right)$ and $(* \mathrm{k} 1)$ or $(* \mathrm{~h} 2)$ correspond to parts of Steps 5, 7, and 9 of Section 4.1 by performing the dynamic and static analysis and the sensitivity analysis, and by finding in (*h1) the $N$ th local optimum, or by finding no solution in $(* \mathrm{~h} 2)$, for the current resonance frequency interval using the IF technique.

Sub-step (*i1) starts a new cycle of the GIF method to search for the local optimum in the next one of the sequential resonance frequency intervals, which corresponds to parts of Steps 6, 7, 8 and 9 of Section 4.1. Sub-step (*i2) corresponds to the last part of Step 7 of Section 4.1, i.e. to start the cycle of the GIF method in the first one $(j=1)$ of the sequential resonance frequency intervals at the right-hand side of the reference resonance frequency interval (RFII), when no solution is found in one of the sequential resonance frequency intervals at the left-hand side of the RFII, or the searching in the interval $\left[\Omega_{0}, \Omega_{1}\right]$ is completed.

Finally, sub-step $(* \mathrm{i} 3)$ corresponds to Step 10 of Section 4.1. Here, the 'best' local optimized solution (with the lowest value of the compliance $C_{d}$ from among the obtained $N$ candidate solutions), is coined as the 'global' optimum solution to the problem.

\section{Examples of topology optimization for minimum dynamic compliance subject to a 'high' excitation frequency using the GIF method}

In this Section, two different numerical examples concerning minimum dynamic compliance design of bi-material platelike structures subject to high excitation frequencies will be validated and illustrated with a view to show in detail how the

GIF method works and to demonstrate the effectiveness and advantages of the method. The upper bound constraint on the static compliance is not considered in this section. As discussed in Section 4.1.1, such a constraint is not imperative here, because the design domain of bi-material plates is fully covered by the stiffer and the softer material, and no risk of 'degeneration' exists.

\subsection{Example 1 - Bi-material square plate-like structure excited by uniformly distributed pressure loading}

This section illustrates the application of the GIF method to the problem of optimizing the topological design of a bi-material, square plate-like structure with clamped edges, see Fig. 6a. A time-harmonic, uniformly distributed transverse external load $p(t)=P \cos \left(\omega_{p} t\right)$ with unit amplitude (i.e. $P=1$, SI units are used throughout this paper) is applied to the upper surface of the plate. The design objective is to minimize the dynamic compliance of the plate for a prescribed 'high' value $\omega_{p}=500$ of the excitation frequency $\omega_{p}$ and a volume fraction of $50 \%$ for the stiffer material $* 1$, and hence for the softer material $* 2$ as well. (For the bi-material model for topology optimization, the reader may refer to Section 2.1.2 and the papers by Bendsøe and Sigmund (1999), Olhoff and Du (2005), and Du and Olhoff (2007a,b)). The stiffer material *1 has the Young's modulus $E^{* 1}=10^{11}$, Poisson's ratio $v=0.3$ and the specific mass $\gamma_{m}^{* 1}=7800$, and the softer material $* 2$ has the properties $E^{* 2}=0.1 E^{* 1}, \gamma_{m}^{* 2}=0.1 \gamma_{m}^{* 1}$, and $v=0.3$. The plate is modeled by 8 -node $3 \mathrm{D}$ isoparametric elements in a $40 \times 40 \times 1 \mathrm{mesh}$, and Wilson incompatible terms (Wilson et al. 1973) are applied to ensure that bending effects are simulated well. Our numerical tests show that the FEM model used in the present paper has very satisfactory element precision and very good convergence for the problem considered in comparison with other models using higher order elements (e.g. 20-node element) and/or a finer mesh (e.g. mesh from $40 \times 40 \times 2$ to $100 \times 100 \times 3$ ).

Fig. 6 Square, bi-material platelike structure $(a=20, b=20, t=1)$ subjected to uniformly distributed harmonic pressure loading on its upper surface. All edges of the plate are clamped. (b) Uniform initial design with the volumetric density $\rho_{e}=0.5$ of the stiffer material. The two materials are evenly mixed in all the elements of the design

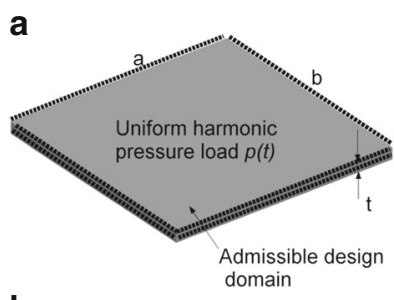

b

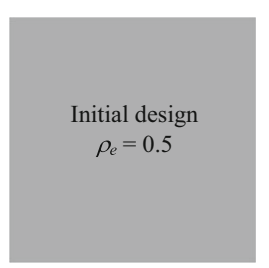


5.1.1 Definition, eigenfrequency analysis, and orthogonality tests of the initial design

Following the computational procedure in Section 4.1 for the GIF method, we in Step 1 choose a uniform bi-material plate as the initial design, cf. Fig. $6 \mathrm{~b}$, and take the materials $* 1$ and $* 2$ to be uniformly mixed in all the elements of the initial design.

In Step 2, we calculate the first 30 eigenfrequencies of free vibrations of the initial design with the fundamental eigenfrequency $\omega_{1}=95.3$ and the $30^{\text {th }}$ eigenfrequency $\omega_{30}=1069.0$ which is anticipated to be well above the prescribed value $\omega_{p}=500$ of the excitation frequency. The orders $i$ and the values of these eigenfrequencies $\omega_{i}$ are shown in the first and second columns of Table 3 in the Appendix.

In Step 3 and Step 4 in Section 4.1, an orthogonality index $\left|\mathbf{P}^{\mathbf{T}} \boldsymbol{\varphi}_{i}\right|$ of the initial design is calculated first, and the results are shown in the fourth column of Table 3 in the Appendix. An empirical error limit $\varepsilon=10^{-6}$ is chosen for the orthogonality tests, i.e. if $\left|\mathbf{P}^{\mathbf{T}} \boldsymbol{\varphi}_{i}\right|<\varepsilon$, the eigenmode $\boldsymbol{\varphi}_{i}$ is regarded to be orthogonal to the loading mode $\mathbf{P}$, and the corresponding eigenfrequency $\omega_{i}$ is identified as a non-resonance frequency; Otherwise, if $\left|\mathbf{P}^{\mathbf{T}} \boldsymbol{\varphi}_{i}\right| \geq \varepsilon$, the eigenmode $\boldsymbol{\varphi}_{i}$ is regarded to be non-orthogonal to the loading mode $\mathbf{P}$, and the corresponding eigenfrequency $\omega_{i}$ is defined as a resonance frequency. Both the vectors $\mathbf{P}$ and $\boldsymbol{\varphi}_{i}$ are normalized to unity before the orthogonality tests. Five resonance frequencies $\Omega_{j}, \quad(j=1, \cdots, 5)$ have been identified successfully within the first 30 eigenfrequencies of the initial design after the orthogonality tests (see the third column of Table 3 in the Appendix). It is found that the prescribed value $\omega_{p}=500$ of the excitation frequency is located between the second and the third resonance frequencies of the initial design, which implies that $m=3$ according to Step 4 . The value of the dynamic compliance $C_{d}$ of the initial design at $\omega=\omega_{p}=500$ is easily obtained by solution of $(1 \mathrm{~b}, \mathrm{c})$, and found to be $C_{d}=6.842 \mathrm{E}-7$.

In order to verify further the results of the orthogonality tests, a series of frequency response analyses of the dynamic compliance of the initial design is performed for unit spacing of the excitation frequencies in the interval $[0$, 1000]. Figure 7 shows the frequency response curve using natural logarithmic coordinates by which the first five resonance frequencies may be identified clearly at about $\Omega_{1}=95, \Omega_{2}=338, \Omega_{3}=542, \Omega_{4}=750$ and $\Omega_{5}=927$. Comparing Fig. 7 with Table 3 in the Appendix, it is verified that the identification of the resonance frequencies by orthogonality tests are accurate and thus may be used as a basis for the following steps. It should be noted that, although the resonance frequencies may be identified in two different ways according to the above discussion, in practice we recommend orthogonality tests as the standard step of identifying the resonance frequencies, since the frequency response analysis over a wide frequency range is normally much more time-consuming.

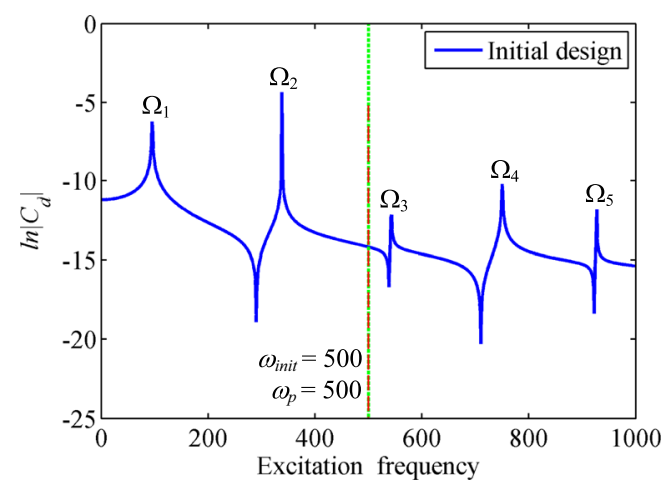

Fig. 7 Dynamic compliance (log. scale) of the initial design (see. Fig. 6b) vs. excitation frequency $\omega$ over the interval $[0,1000]$. Five resonance frequencies may be identified clearly $\left(\Omega_{1}=95, \Omega_{2}=338, \Omega_{3}=542\right.$, $\Omega_{4}=750$, and $\left.\Omega_{5}=927\right)$. The prescribed value $\omega_{p}=500$ of the excitation frequency is seen to be located between the second and the third resonance frequencies

\subsubsection{Cases of searching local optimized designs in different resonance frequency intervals embracing the prescribed excitation frequency}

It is now possible to start the design process in some pertinent ways by locating the initial value $\omega_{\text {init }}$ of the excitation frequency $\omega$ in different intervals between adjacent resonance frequencies of the initial design (cf. the preceding section and Table 3 in the Appendix), and in cases apply the IF technique (Section 2.4) to change the values and orders of the resonance frequencies embracing the prescribed excitation frequency $\omega_{p}$.

In the following, we will use Case A, B, C and D to designate four different design cases corresponding to four different ways of starting the design process and the corresponding sequential solution strategies. As references, the results of the eigenfrequency analysis and the orthogonality tests of the initial design are given in Table 3 in the Appendix, and the results corresponding to each of the four design cases are given in Tables 4, 5, 6, and 7 in the Appendix.

Case A. $\omega_{\text {init }}=500 \rightarrow \omega_{\text {final }}=\omega_{p}=500$

Following Step 5 in Section 4.1, we first consider the case of starting the design process with the initial value $\omega=500$ of the excitation frequency, which is found to be located between the second and the third resonance frequencies (i.e. $\omega \in\left[\Omega_{2}\right.$, $\left.\Omega_{3}\right]$ ) of the initial design, cf. Table 3 in the Appendix . Since the initial value of the excitation frequency is the same as the prescribed value $\omega_{p}=500$ for the optimized design, no incrementation of the excitation frequency $\omega$ is applied in this case. Hereby the 'conventional' topology optimization based on iterative solution of (1a-e) for minimization of the dynamic compliance of the forced vibrating bi-material plate is performed for the given amplitude and spatial distribution of the external dynamic loading subject to the fixed, prescribed value $\omega=\omega_{p}=500$ of the excitation frequency. The iterations are started out from the initial design chosen in Step 1 and its frequency response curve is depicted in Fig. 7. 
The optimized topology obtained in Case A is illustrated in Fig. 8a. Comparison of the frequency response curves of the dynamic compliance of the initial design (see Fig. 6b) and the optimized design (Fig. 8a) is shown in Fig. 8b and c. Here, it is seen that for the optimized design, the excitation frequency is located in the interval between the second resonance frequency $\Omega_{2}^{o p t}=300$ and the third resonance frequency $\Omega_{3}^{o p t}=533$, and
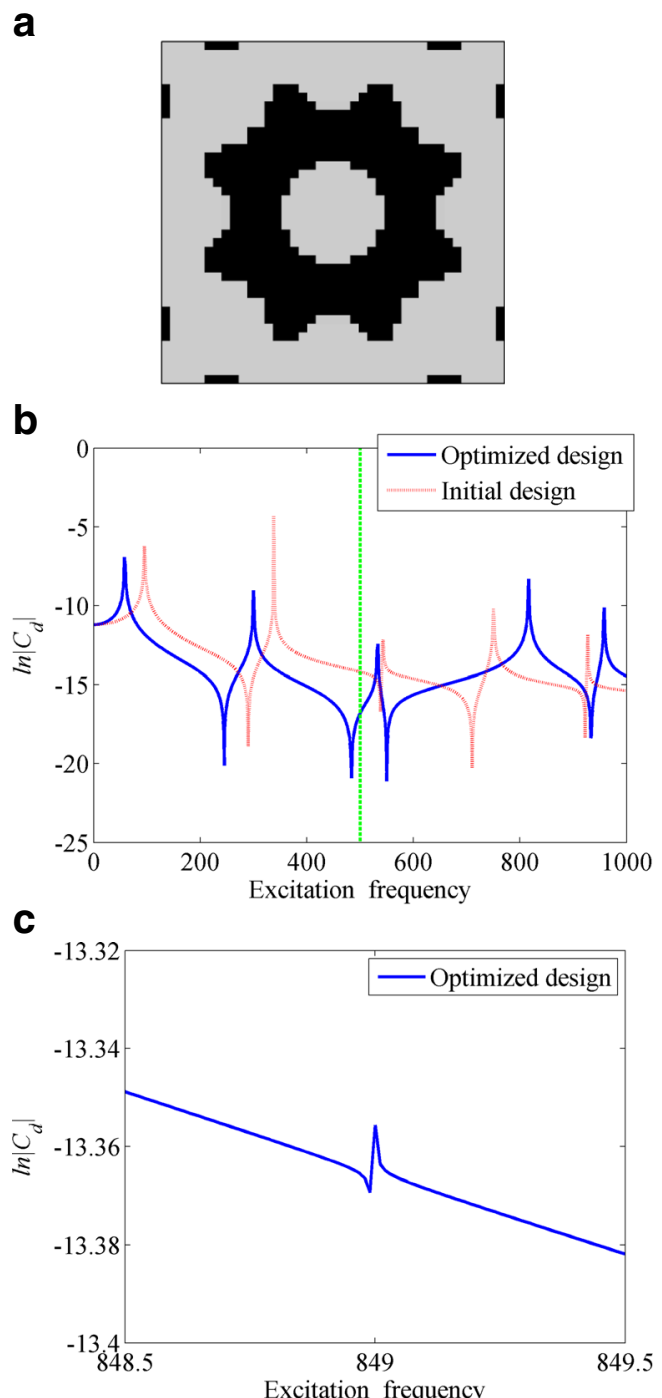

Fig. 8 Case A: (a) Optimized topology of the forced vibrating bimaterial plate, where the prescribed value $\omega_{p}=500$ of the excitation frequency is located between the second and the third resonance frequencies of the optimized design (i.e. $\omega_{p} \in\left[\Omega_{2}^{o p t}, \Omega_{3}^{o p t}\right]$ ). (b) Comparison of the frequency response curves of the dynamic compliance of the initial design (see Fig. 6b) and the optimized design (see Fig. 8a). It can be seen that the prescribed value $\omega_{p}=500$ of the excitation frequency is located between the second and the third resonance frequencies, for both the initial and the optimized design (cf. also Tables 3 and 4 in the Appendix). (c) A resonance frequency at about $\Omega_{5}=849$ of the optimized design may be identified by a close look on the frequency response curve in addition to the five resonance frequencies identified directly from the frequency response curve of the optimized design in Fig. 8 b, i.e. $\Omega_{1}=58, \Omega_{2}=300, \Omega_{3}=533, \Omega_{4}=816$, and $\Omega_{6}=958$ (refer also to Table 4 in the Appendix) the value of the minimized dynamic compliance $C_{d}$ of the local optimized design for $\omega_{p}=500$ is found to be $C_{d}=5.013 \mathrm{E}-8$ (see also Table 4 in the Appendix). This implies that the prescribed excitation frequency for the optimized design is located in an interval with the same orders of the limiting resonance frequencies (i.e. $\omega_{p} \in\left[\Omega_{2}^{o p t}, \Omega_{3}^{o p t}\right]$ ) as those of the initial design (i.e. $\left.\omega_{\text {init }} \in\left[\Omega_{2}^{I D}, \Omega_{3}^{I D}\right]\right)$.

The reader is referred to the third paragraph of Section 4 where the physical background for this result is explained.

Case B. $\omega_{\text {init }}=337.00 \rightarrow \omega_{\text {final }}=480.5<\omega_{p}=500$

Following Step 6 in Section 4.1, we set $i=m-1=2$, and then perform Step 7, i.e. an IF technique for the initial design in Fig. 6b with an initial value $\omega=337$ of the excitation frequency that is slightly smaller than the resonance frequency $\Omega_{2}=338$ of the initial design (see Fig. 9 and Table 3 in the Appendix). According to the IF technique described in Section 2.4, Case B consists in solving incrementally the topology design problem (1a-e) for minimum dynamic compliance subject to increasing $\omega$ in small increments towards its originally prescribed value $\omega_{p}$, using as input for each new increment the topology design and displacement amplitudes obtained in the preceding increment.

Please bear in mind that in order to increase the excitation frequency $\omega$ (and hence the second resonance frequency $\Omega_{2}$ ), then $\omega$ should in each increment be assigned a value at the near left-hand side of the resonance frequency $\Omega_{2}$ of the current design with a view to 'push' the second resonance frequency $\Omega_{2}$ to move in the right-hand direction of the frequency axis (cf. Fig. 9). Thus, it is necessary to identify the second resonance frequency for each step of the incremented frequency, which is enabled by performing the eigenfrequency analysis and orthogonality tests in Table 5 in the Appendix.

It is also worth mentioning that in the early stage of the design procedure, the resonance frequency nearest to the excitation frequency $\omega$ will normally move away from $\omega$ in comparatively large increments, and thereby quickly decrease the dynamic compliance to be minimized. Thus, if we carefully control the

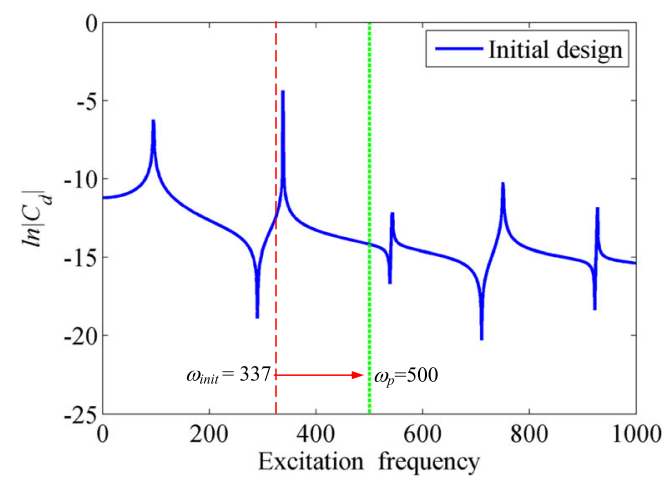

Fig. 9 Case B: Dynamic compliance (log. scale) of the initial design (see Fig. 6b) vs. excitation frequency $\omega$ over the interval $[0,1000]$. The design process is started using the initial value $\omega=337$ of the excitation frequency that is slightly smaller than the second resonance frequency $\Omega_{2}=338$ of the initial design 
change of the excitation frequency $\omega$ and initially use a smaller value of the increment of $\omega$, then there is normally no risk that the excitation frequency will 'jump over' the adjacent resonance frequency, and the eigenfrequency analysis and the orthogonality tests may be skipped in the early stage of the incremental design procedure in order to save computation time.

The process of increasing the excitation frequency $\omega$ in the above incremental design procedure will be stopped when any of the following two conditions is satisfied: (1) the excitation frequency $\omega$ reaches the prescribed value $\omega_{p}=500$; (2) the second resonance frequency $\Omega_{2}$ reaches its maximum value that is less than the prescribed value $\omega_{p}=500$ of the excitation frequency for the prescribed loading mode (uniform pressure loading). If condition (1) is satisfied firstly, then a valid local optimized solution has been found for the case that the excitation frequency is located in the interval between the first and the second resonance frequencies, and the solution may be considered as a candidate for the 'global optimized solution' of the current problem. If condition (2) is satisfied firstly, it implies that no valid solution can be found for the case that the excitation frequency is located in the interval between the resonance frequencies with the orders considered.

In our computations on Case B of the current example, it is found that when the second resonance frequency $\Omega_{2}$ is 'pushed' to the value about 481.5 (see also Table 5 in the Appendix), it cannot be increased further towards the prescribed value $\omega_{p}=500$ by the IF technique, and this implies that the solution set of the design Case B is a null set.

Although there is no valid solution obtained in Case B, as a reference, the final topology obtained in Case B corresponding to the value $\omega_{\text {final }}=480.5$ of the excitation frequency is shown in Fig. 10a. The frequency response curve for the dynamic compliance of the final reference design in Fig. 10a is shown in Fig. 10b, and the second resonance frequency reaches its maximum value 481.5 for the prescribed loading mode of the current example (see also Table 5 in the Appendix).

As the next step, since the excitation frequency $\omega$ cannot reach the prescribed value $\omega_{p}=500$ from the left-hand side, when $\omega_{p}$ is located in the interval $\left[\Omega_{1}, \Omega_{2}\right]$ between the first and second resonance frequencies, we will jump to Step 8 and 9 according to Step 7 in Section 4.1, and move the excitation frequency $\omega$ from the right-hand side of the prescribed value $\omega_{p}=500$, and see if we can find local optimized solutions of problem (1a-e) located in intervals between resonance frequencies of higher orders than those considered above. The details will be discussed in Cases $\mathrm{C}$ and $\mathrm{D}$.

Case C. $\omega_{\text {init }}=545 \rightarrow \omega_{\text {final }}=\omega_{p}=500$

Following Step 8 in Section 4.1, we set $i=m=3$, and then perform Step 9, i.e. the IF technique (Section 2.4) with the initial design shown in Fig. $6 \mathrm{~b}$ and an initial value $\omega=545$ of the excitation frequency that is slightly larger than the resonance frequency $\Omega_{3}=542.5$ of the initial design (see Fig. 11 and Table 3 in the Appendix). The operation in Step 9 is quite a

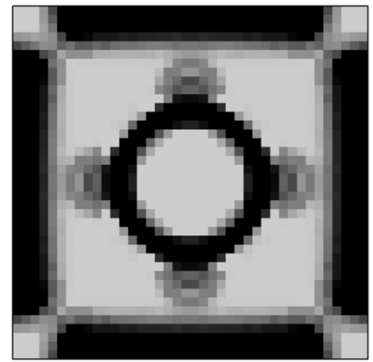

b

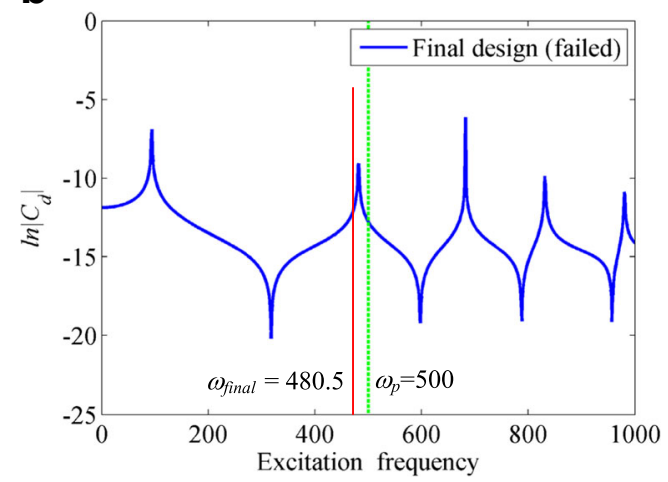

Fig. 10 Case B: (a) Final reference topology of the forced vibrating bimaterial plate, where the excitation frequency has the value $\omega_{\text {final }}=480.5$ and cannot reach the prescribed value $\omega_{p}=500$ when it is located in the interval between the first and the second resonance frequencies of the initial design (i.e. $\omega \in\left[\Omega_{1}, \Omega_{2}\right]$ ). (b) Frequency response curve of the dynamic compliance of the final reference design (see Fig. 10a). The excitation frequency $\omega$ cannot reach the prescribed value $\omega_{p}=500$, which implies that the solution set of the Case B is a null set (see also Table 5 in the Appendix)

similar to that in Step 7 except for that now the excitation frequency $\omega$ is moved towards its prescribed value $\omega_{p}=500$ from the right-hand side of the frequency axis, and in each incremental step of the IF technique, the value of $\omega$ should be set slightly larger than the third resonance frequency, until $\omega$ successfully reaches the prescribed value $\omega_{p}=500$.

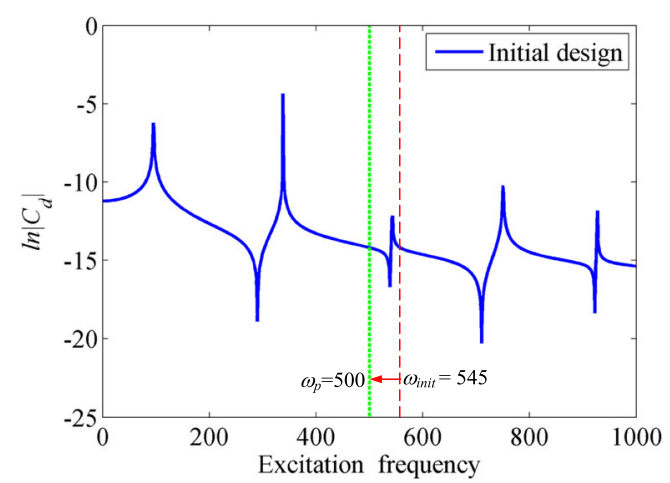

Fig. 11 Case C: Dynamic compliance (log. scale) of the initial design in Fig. 6 b vs. excitation frequency $\omega$ over the interval $[0,1000]$. The design procedure is started using the initial value $\omega=545$ of the excitation frequency that is slightly larger than the third resonance frequency $\Omega_{3}=542.5$ of the initial design 
The optimized topology obtained in Case $\mathrm{C}$ is shown in Fig. 12a, and a comparison of the dynamic compliance curves for the initial design in Fig. $6 \mathrm{~b}$ and the optimized design in Fig. 12a is made in Fig. 12b and c. As is shown for the local optimized design in Table 6 in the Appendix, the prescribed excitation frequency $\omega_{p}=500$ is located in the interval between the third resonance frequency $\Omega_{3}^{o p t}=462.0$ and the fourth resonance frequency $\Omega_{4}^{o p t}=712.45$, (i.e. $\omega_{p} \in\left[\Omega_{3}^{o p t}\right.$,

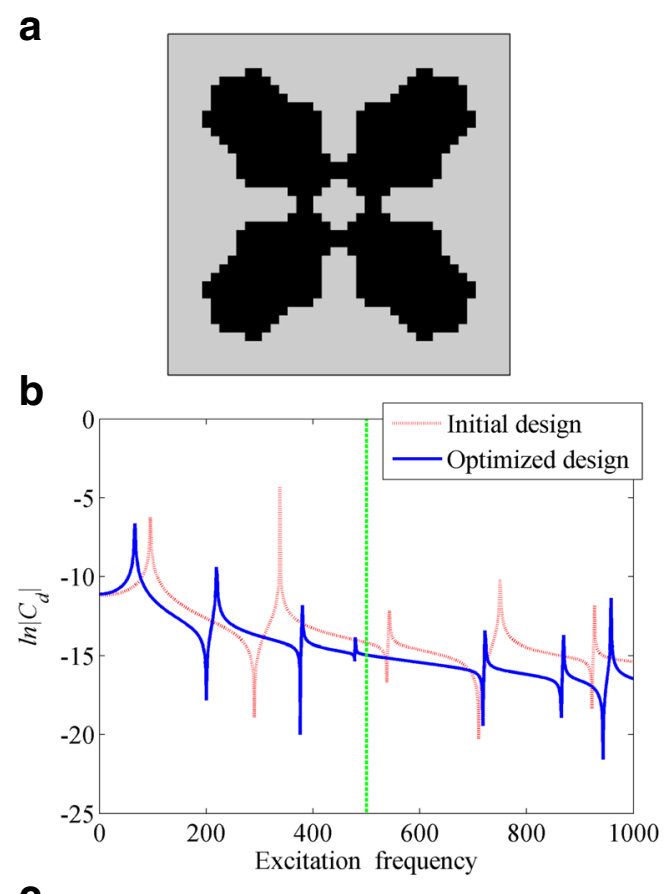

C

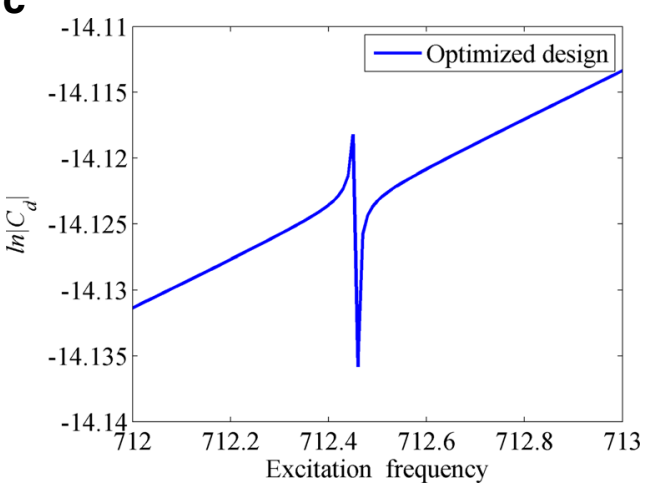

Fig. 12 Case C: (a) Optimized topology of the forced vibrating bimaterial plate, where the value $\omega_{p}=500$ of the prescribed excitation frequency is located between the third and the fourth resonance frequencies of the local optimized design (i.e. $\omega_{p} \in\left[\Omega_{3}^{o p t}, \Omega_{4}^{o p t}\right]$ ). (b) Comparison of the dynamic compliance curves of the initial design in Fig. $6 \mathrm{~b}$ and the optimized design in Fig. 12a. It is seen that the prescribed value $\omega_{p}=500$ of the excitation frequency is located in the interval between the third and the fourth resonance frequencies of the local optimized design (cf. also Table 6 in the Appendix). (c) A resonance frequency at about $\Omega_{4}=712.45$ of the optimized design may be identified by a close look on the frequency response curve in addition to the five resonance frequencies identified directly from the frequency response curve of the optimum design in Fig. 12b, cf. Table 6 in the Appendix
$\left.\Omega_{4}^{o p t}\right]$ ), and the value of the minimized dynamic compliance $C_{d}$ of the local optimized design is found to be $C_{d}=6.424 \mathrm{e}-10$.

The fact that the excitation frequency $\omega$ reaches its prescribed value $\omega_{p}=500$ by means of the IF technique implies that a valid local optimized solution has been found in the present case where the excitation frequency is located in the interval between the third and fourth resonance frequencies, and the solution may thus be considered as another candidate for the 'global optimized solution'.

As the final part of Step 9, we set the counter of local optimized solutions $N=N+1$, repeat Step 9 with $i=i+1$, and try to search yet another local optimized solution in the subsequent interval between resonance frequencies of higher orders. This will be discussed in Case D.

Case D. $\omega_{\text {init }}=752 \rightarrow \omega_{\text {final }}=\omega_{p}=500$

Following a very similar approach as in Step 9 for Case C, another valid local optimized solution is obtained by IF technique for $\omega_{p}=500$ located in the interval between the fourth and fifth resonance frequencies, i.e. $\omega_{p} \in\left[\Omega_{4}^{o p t}, \Omega_{5}^{o p t}\right]$, and the solution is associated with the minimized dynamic compliance $C_{d}=3.216 \mathrm{e}-7$, see Table 7 in the Appendix). The corresponding results are shown in Fig. 13.

\section{a}

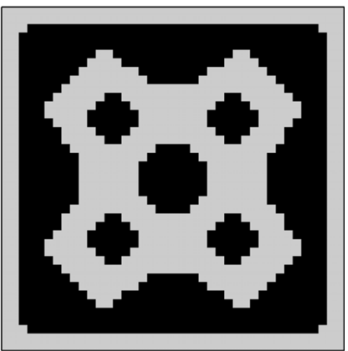

b

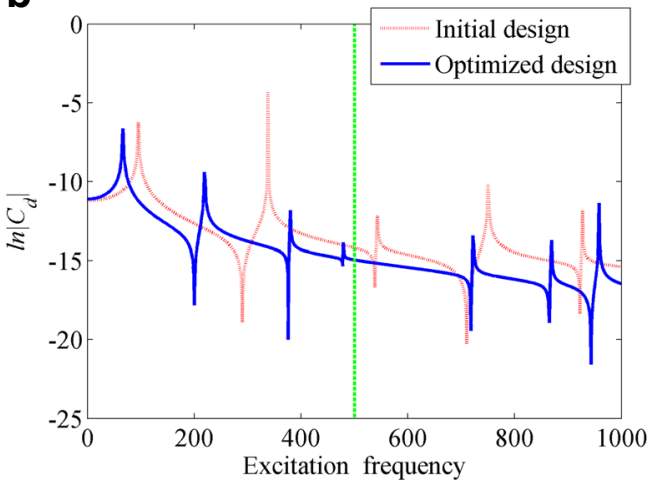

Fig. 13 Case D: (a) Optimized topology of the forced vibrating bimaterial plate, where the value $\omega_{p}=500$ of the excitation frequency is located between the fourth and the fifth resonance frequencies of the optimized design (i.e. $\omega_{p} \in\left[\Omega_{4}^{o p t}, \Omega_{5}^{o p t}\right]$ ). (b) Comparison of the frequency response curves of the dynamic compliance of the initial design (see Fig. 6b) and the optimized topology (see Fig. 13a). It is seen that the prescribed value $\omega_{p}=500$ of the excitation frequency is located in the intervals between the 2 th and 3 rd resonance frequencies of the initial design, and the 4th and 5 th resonance frequencies of the optimized design (cf. Tables 3 and 7, respectively, in the Appendix) 
Table 1 Comparison of the three valid local optimized solutions obtained for the excitation frequency $\omega_{p}=500$ by the 'generalized incremental frequency method' (GIF method)

\begin{tabular}{|c|c|c|c|c|}
\hline Case & $\begin{array}{l}\text { Dynamic compliance } \\
\text { of the initial design } \\
\left(\omega_{p}=500\right)\end{array}$ & $\begin{array}{c}\text { Dynamic compliance } \\
\text { of the optimized } \\
\text { design }\left(\omega_{p}=500\right) \\
\end{array}$ & $\begin{array}{c}\text { Optimized } \\
\text { topology }\end{array}$ & $\begin{array}{c}\text { Interval of } \\
\text { resonance } \\
\text { frequencies for } \omega_{p} \\
\end{array}$ \\
\hline A & $6.842 \mathrm{E}-7$ & $5.013 \mathrm{E}-8$ & & $\omega_{p} \in\left[\Omega_{2}^{o p t}, \Omega_{3}^{o p t}\right]$ \\
\hline $\mathrm{C}$ & $6.842 \mathrm{E}-7$ & $6.424 E-10$ & & $\omega_{p} \in\left[\Omega_{3}^{o p t}, \Omega_{4}^{o p t}\right]$ \\
\hline $\mathrm{D}$ & $6.842 \mathrm{E}-7$ & $3.216 \mathrm{E}-7$ & & $\omega_{p} \in\left[\Omega_{4}^{o p t}, \Omega_{5}^{o p t}\right]$ \\
\hline
\end{tabular}

Since the excitation frequency reaches the prescribed value $\omega_{p}=500$ successfully, we repeat the operation in the last paragraph of Case C, i.e. we set the counter of local optimum solutions $N=N+1$, and repeat Step 9 with $i=i+1$ to search for a local optimized solution in the next interval between the consecutive fifth and sixth resonance frequencies. However, we find by our sequential computation that the excitation frequency $\omega$ cannot reach the prescribed value $C_{d}$ successfully from its initial value that was taken to be slightly higher than that of the fifth resonance frequency $\Omega_{5}=926.83$ of the initial design, cf. Table 3 in the Appendix.

\subsubsection{Results of example 1}

We finally move to Step 10 of the GIF method developed in Section 4 and outlined in Section 4.1 to complete the overall design optimization problem by selecting the 'global optimized solution' from among the valid candidate solutions obtained in Cases A, C and D. The minimized values of the dynamic compliances $C_{d}$ obtained by the three valid local topology optimizations performed in Cases $\mathrm{A}, \mathrm{C}$ and $\mathrm{D}$ are listed in Table 1. Here, the topology design obtained in Case C and shown in the fourth column of Table 1 is associated with the smallest value $C_{d}=6.424 \mathrm{E}-10$ of the minimized dynamic compliances listed in the third column. Thus, the results of Case $C$ may be regarded as the 'global optimized solution' of the problem in Example 1.

The results of the optimizations in the Cases A, C and D are summarized in the third to fifth columns of Table 1, and are all based on the same initial design defined and studied in Section 5.1.1. For the initial design, the excitation frequency $\omega_{p}=500$ is located in the interval between the $2^{\text {nd }}$ and the $3^{\text {rd }}$ resonance frequencies, i.e. $\omega_{p} \in\left[\Omega_{2}^{I D}, \Omega_{3}^{I D}\right]$, and the corresponding dynamic compliance of the initial design is calculated to be $C_{d}=6.842 \mathrm{E}-7$. This value may be considered as a reference for the minimized compliance values listed for the Cases A, C and $\mathrm{D}$ in the third column of Table 1.

The local design problem in Case A corresponds precisely to Step 5 in the computational procedure of the GIF method in Section 4.1, and consists in iterative solution of the 'conventional formulation' in (1a-e) of the topology optimization problem subject to the given, constant value $\omega=\omega_{p}=500$, while the local design problems in Cases $\mathrm{C}$ and $\mathrm{D}$ are solved using the IF technique defined in Section 2.4. The fact that the dynamic compliance of the optimized design is much smaller in Case C than in Case A confirms that for prescribed 'high' excitation frequencies, the 'conventional formulation' for optimum design in 1(a-e) may not lead to the appropriate solution.

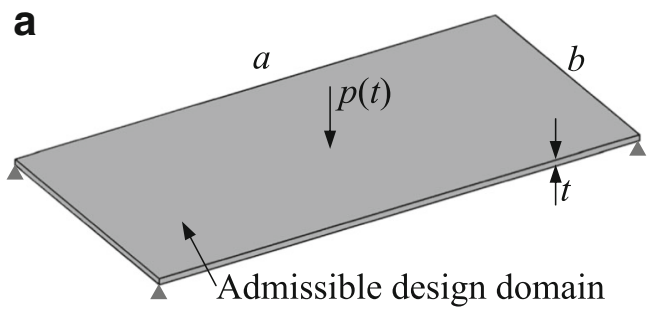

b

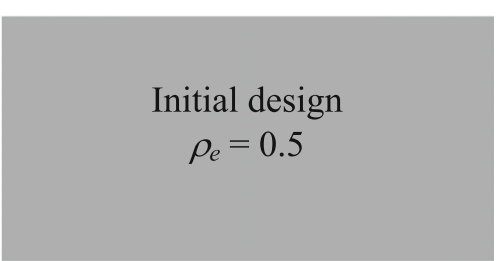

Fig. 14 (a) Bi-material rectangular plate-like structure $(a=2, b=1$, $\mathrm{t}=0.025)$ subjected to a concentrated harmonic transverse force at the center of its upper surface. The plate is simply supported at its four corners. (b) Uniform initial design with the volumetric density $\rho_{e}=0.5$ of the stiffer material 


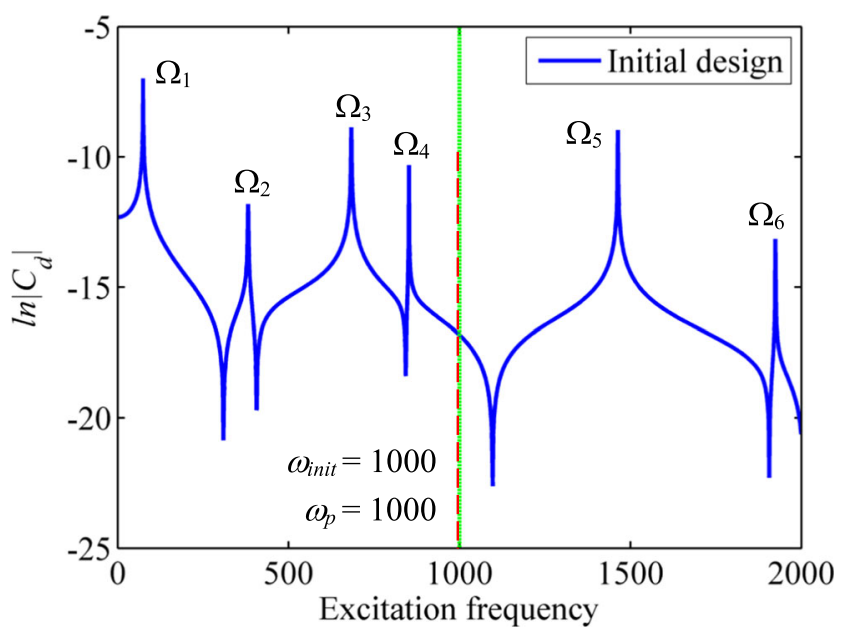

Fig. 15 Case A: Dynamic compliance (log. scale) of the initial design (Fig. 14b) vs. excitation frequency $\omega$ over the interval $[0,2000]$. Six resonance frequencies may be identified clearly $\left(\Omega_{1}=74, \Omega_{2}=381\right.$, $\Omega_{3}=683, \Omega_{4}=852, \Omega_{5}=1463$, and $\left.\Omega_{6}=1924\right)$. The prescribed value $\omega_{p}=1000$ of the excitation frequency is located between the 4th and 5 th resonance frequencies, i.e. $\omega_{p} \in\left[\Omega_{4}, \Omega_{5}\right]$. The design process in Case $\mathrm{A}$ corresponds to Step 5 of the GIF method, and is started using the value $\omega_{\text {init }}=1000$ of the excitation frequency $\omega$, and keeping $\omega$ fixed at $\omega=\omega_{p}=1000$ during iterative solution of the 'conventional' (1a-e) to convergence

\subsection{Example 2 - Bi-material rectangular plate-like structure excited by concentrated transverse force at the center}

This example concerns topological design of a rectangular, corner-supported, bi-material plate-like structure (see Fig. 14), using the 'generalized incremental frequency method' (GIF method). A time-harmonic, concentrated transverse external load $p(t)=P \cos \left(\omega_{p} t\right)$ with $P=1$ (SI units are used throughout), is applied to the center of the upper surface of the plate. The design objective is to minimize the dynamic compliance of the plate for a prescribed 'high' value of the excitation frequency $\omega_{p}$ and a volume fraction of $50 \%$ for the given stiffer material $* 1$, which has the Young's modulus $E^{* 1}=10^{11}$, Poisson's ratio $v=0.3$ and the specific mass $\gamma_{m}^{* 1}=7800$. The soft material $* 2$ has the properties $E^{* 2}=0.1 E^{* 1}, \gamma_{m}^{* 2}=0.1 \gamma_{m}^{* 1}$, and $v=0.3$. The admissible design domain of the plate is divided into a $80 \times 40 \times 1$ mesh with 3D 8-node isoparametric elements, and Wilson incompatible terms (Wilson et al. 1973) are applied to ensure that bending effects are simulated well. A geometry symmetry constraint is introduced during the design process for manufacturing convenience. Additional penalization terms are applied to the design objective function in order to obtain clear 0-1 topology results (Du and Olhoff 2010). The (high) value of the excitation frequency considered in this example is $\omega_{p}=1000$.

Figure 15 shows the frequency response curve of the uniform initial design in Fig. 14b over the interval $[0,2000]$ of the excitation frequency $\omega$, from which six resonance frequencies may be identified, i.e. $\Omega_{1}=74, \Omega_{2}=381, \Omega_{3}=683, \Omega_{4}=852, \Omega_{5}=1463$, and $\Omega_{6}=1924$. These resonance frequencies correspond to the eigenfrequencies $\omega_{1}, \omega_{4}, \omega_{7}, \omega_{9}, \omega_{15}$, and $\omega_{19}$ of the initial design. The prescribed value $\omega_{p}=1000$ of the excitation frequency is seen to be located in the interval between the 4th and 5th resonance frequencies, i.e. $\omega_{p} \in\left[\Omega_{4}, \Omega_{5}\right]$.

Following the computational scheme of the GIF method in Section 4.1, we performed six design cases starting from six different initial values $\omega_{\text {init }}$ of excitation frequencies located in different intervals between the resonance frequencies, i.e.

Case A: $\omega_{\text {init }}=1000 \in\left[\Omega_{4}, \Omega_{5}\right]$;

Case B1: $\omega_{\text {init }}=850.9 \in\left[\Omega_{3}, \Omega_{4}\right]$;

Case B2: $\omega_{\text {init }}=682.3 \in\left[\Omega_{2}, \Omega_{3}\right]$;

Case B3: $\omega_{\text {init }}=380.4 \in\left[\Omega_{1}, \Omega_{2}\right]$;

Case C1: $\omega_{\text {init }}=1464.2 \in\left[\Omega_{5}, \Omega_{6}\right]$;

Case $\mathrm{C} 2: \omega_{\text {init }}=1925.1 \in\left[\Omega_{6}, \Omega_{7}\right]$. (a)

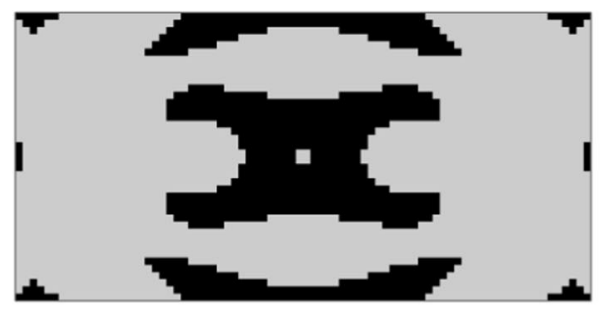

Fig. 16 Case A: (a) Optimized topology of the forced vibrating bimaterial plate, where the prescribed value $\omega_{p}=1000$ of the excitation frequency is located between the 4 th and 5 th resonance frequencies of the optimized design (i.e. $\omega_{p} \in\left[\Omega_{4}^{o p t}, \Omega_{5}^{o p t}\right]$ ). (b) Comparison of the dynamic compliance curves for the initial design (Fig. 14b) and the (b)

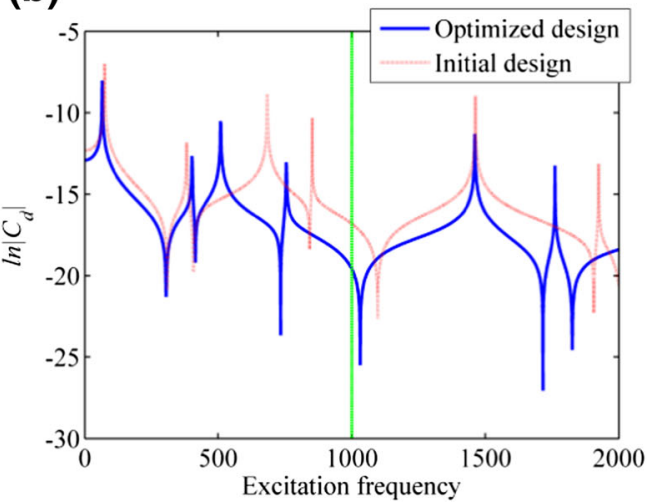

optimized design (Fig. 16a). It is seen that the prescribed value $\omega_{p}=1000$ of the excitation frequency is located in intervals between the 4th and the 5th resonance frequencies for both the initial and the optimized designs. Note that this finding is supported by the more general result developed in the third paragraph of Section 4 
(a)

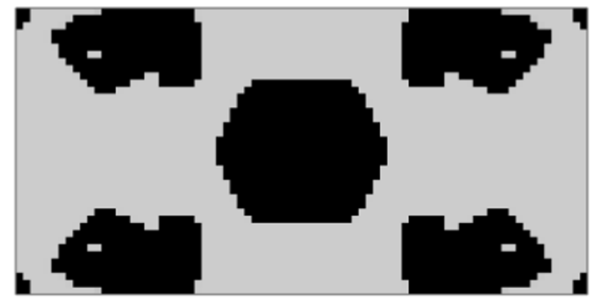

Fig. 17 Case B1: (a) Optimized topology of the forced vibrating bimaterial plate, where the prescribed value $\omega_{p}=1000$ of the excitation frequency is located between the 3 th and the 4 th resonance frequencies of the optimized design (i.e. $\omega_{p} \in\left[\Omega_{3}^{o p t}, \Omega_{4}^{o p t}\right]$ ). (b) Comparison of the dynamic compliance curves for the initial design (Fig. 14b) and the

The design results are shown in Fig. 16 for Case A; Fig. 17 for Case B1; Fig. 18 for Case B2; Fig. 19 for Case B3; Fig. 20 for Case C1; and in Fig. 21 for Case C2. Here, four valid locally optimized solutions were identified in the Cases A, $\mathrm{B} 1, \mathrm{~B} 2$ and $\mathrm{C} 1$ by using the GIF method. A summary of these design results and the selection of the global optimized solution of the present problem are presented in Table 2.

\subsubsection{Results of example 2}

Four valid local optimized solutions were identified in the Cases A, B1, B2 and C1 of Example 2 by using the GIF method. A summary of the design results and the selection of the 'global optimized solution' of the current example are presented in Table 2. Here, it can be seen that the design obtained (b)

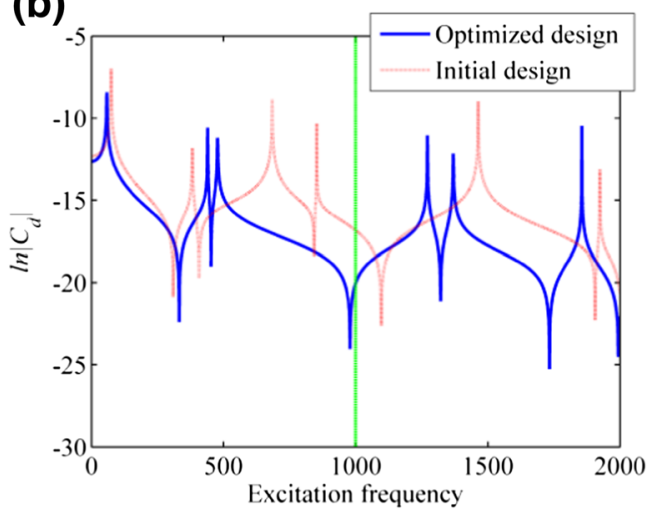

optimized design (Fig. 17a). It is seen that the prescribed value $\omega_{p}=1000$ of the excitation frequency is located in intervals with different orders of the resonance frequencies for the initial design and the optimized design, i.e. $\omega_{p} \in\left[\Omega_{5}^{I D}, \Omega_{5}^{I D}\right]$ and $\omega_{p} \in\left[\Omega_{3}^{o p t}, \Omega_{4}^{o p t}\right]$, respectively

in Case B1 has the smallest value of the minimized dynamic compliance, i.e. $C_{d}=1.970 \mathrm{E}-9$. It is therefore reasonable to consider the local optimized design obtained in Case B1 to be the 'global optimized solution' of the present problem.

All in all, the results summarized in Table 2 exhibit characteristics that are analogous to those in Table 1 (cf. Section 5.1.3), and analogous remarks can be made.

\section{Summary}

Problems of structural topology optimization with the objective of minimizing the dynamic compliance (maximizing the integral dynamic stiffness) of single- and bi-material continuum structures subjected to forced, time-harmonic vibration (a)

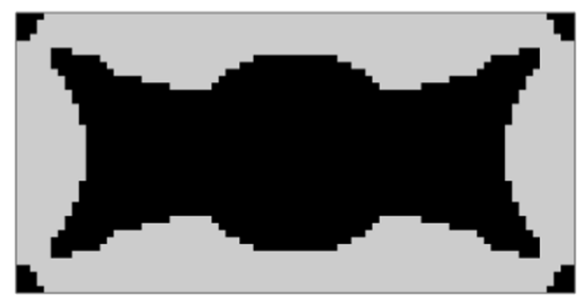

Fig. 18 Case B2: (a) Optimized topology of the forced vibrating bimaterial plate, where the prescribed value $\omega_{p}=1000$ of the excitation frequency is located between the 2 nd and 3rd resonance frequencies of the optimized design. (b) Comparison of the dynamic compliance curves of the initial design (Fig. 14b) and the optimized design (Fig. 18a). It is (b)

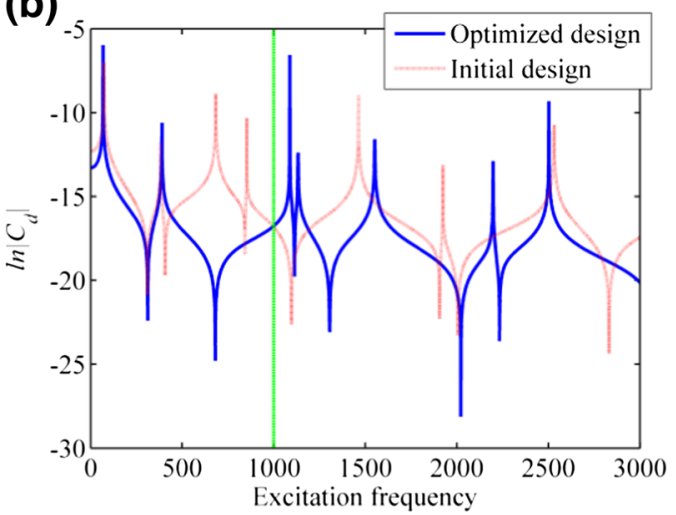

seen that the prescribed value $\omega_{p}=1000$ of the excitation frequency is located in intervals with different orders of the resonance frequencies for the initial design and the optimized design (i.e. $\omega_{p} \in\left[\Omega_{4}^{I D}, \Omega_{5}^{I D}\right]$ and $\left.\omega_{p} \in\left[\Omega_{2}^{o p t}, \Omega_{3}^{o p t}\right]\right)$ 
(a)

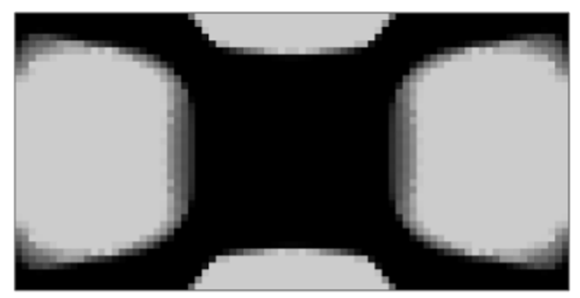

Fig. 19 Case B3 (failed case): (a) Final reference topology of the forced vibrating bi-material plate, where the excitation frequency has the value $\omega_{\text {final }}=562.2$ and cannot reach the prescribed value $\omega_{p}=1000$ when it is located in the interval between the 1 st and the 2 nd resonance frequencies,

with prescribed external excitation frequency, amplitude and spatial distribution of the dynamic loading, are studied in this paper. Excitation frequencies with any prescribed positive value are considered.

It is shown in the paper that the design objective of the forced vibration problem may be implemented along different design paths according to different levels of the external excitation frequency $\omega$. In cases where the harmonic loading is associated with an initial value $\omega=\omega_{\text {init }}$ of the excitation frequency that is less than the fundamental resonance frequency of an initial design, the minimum dynamic compliance design process may be driven by the so-called 'incremental frequency technique' (IF technique) presented in this paper, whereby the excitation frequency $\omega$, and, at the same time, the fundamental resonance frequency $\Omega_{1}$ may be increased sequentially up to a prescribed final value, $\omega_{\text {final }}$. This procedure delivers the

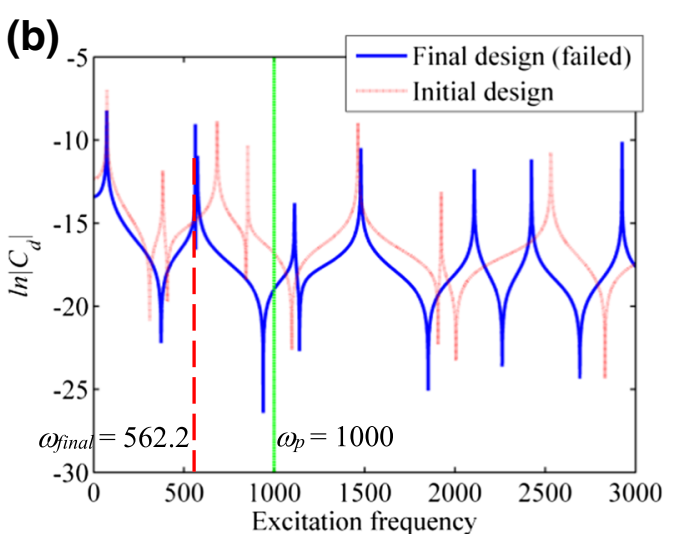

i.e. $\omega \in\left[\Omega_{1}, \Omega_{2}\right]$. (b) Comparison of the dynamic compliance curves for the initial design (Fig. 14b) and the final reference design (Fig. 19a). The excitation frequency $\omega$ cannot reach the prescribed value $\omega_{p}=1000$, which implies that the solution set of the design Case B3 is a null set

desirable result that the optimized structure is associated with minimum dynamic compliance subject to the prescribed final excitation frequency, and also implies an effective improvement (decrease) of the static compliance of the structure.

Based on the IF technique we have in the present paper developed the so-called 'generalized incremental frequency method' (GIF method) with applicability for any prescribed high or low value of the external excitation frequency. The GIF method provides a way of searching for different local optimized solutions in a systematic manner in different disjointed resonance frequency sub-intervals, and hereby the 'global' optimum solution of the problem may be identified from among a small number of obtained candidate local optimum solutions.

In the paper, a computational procedure for the GIF method is developed for determining the 'global' optimum topological design associated with minimum dynamic compliance. This

(a)

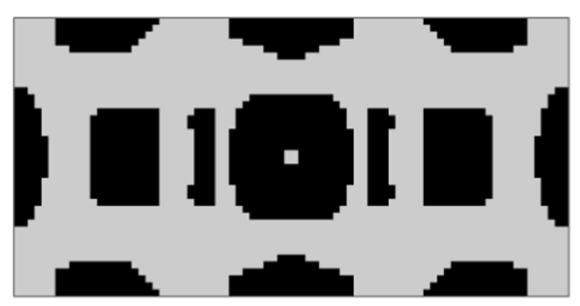

Fig. 20 Case C1: (a) Optimized topology of the forced vibrating bimaterial plate, where the prescribed value $\omega_{p}=1000$ of the excitation frequency is located between the 5 th and the 6 th resonance frequencies of the final optimized design. (b) Comparison of the dynamic compliance curves for the initial design (Fig. 14b) and the optimized design (b)

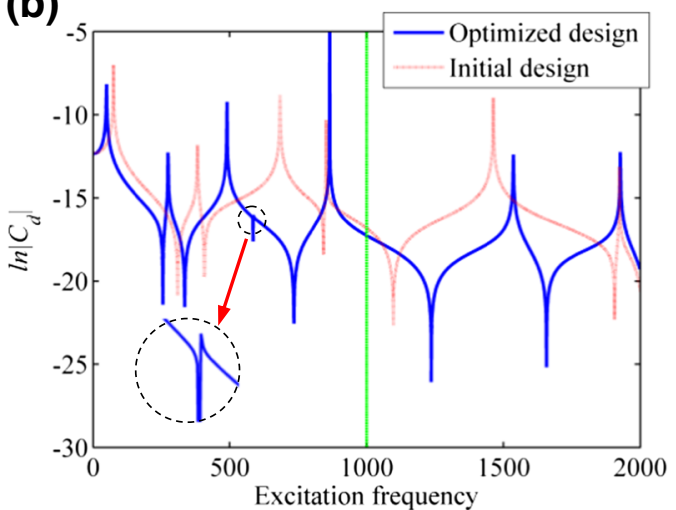

(Fig. 20a). It is seen that the prescribed value $\omega_{p}=1000$ of the excitation frequency is located in intervals with different orders of the resonance frequencies for the initial design and the optimized design (i.e. $\omega_{p} \in\left[\Omega_{4}^{I D}, \Omega_{5}^{I D}\right]$ and $\left.\omega_{p} \in\left[\Omega_{5}^{o p t}, \Omega_{6}^{o p t}\right]\right)$ 
(a)

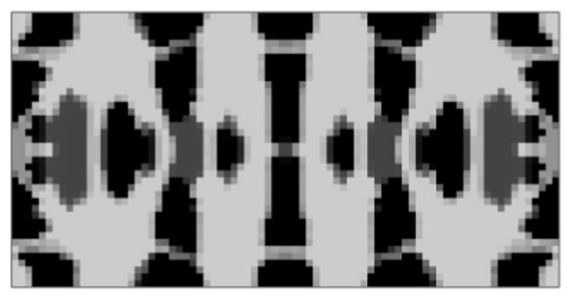

Fig. 21 Case C2 (failed case): (a) Final reference topology of the forced vibrating bi-material plate, where the excitation frequency has the value $\omega_{\text {final }}=1331.4$ and cannot reach the prescribed value $\omega_{p}=1000$ when it is located in the interval between the 6 th and the 7 th resonance frequencies,

procedure is a single, unified algorithm for handling of both 'low' and 'high' excitation frequencies, as well as single- and bi-material structures.

Examples of a single-material structure with a 'low' excitation frequency, and two bi-material structures with 'high' excitation frequencies have been successfully solved, and have validated the algorithm.

With a view to study numerically the limiting behaviour of a design in the vicinity of vanishing excitation frequency $\omega=0$, the IF technique was applied for minimization of the dynamic compliance along a path of decreasing sequentially the excitation frequency $\omega$, and, at the same time, the fundamental resonance frequency $\Omega_{1}$ to a final value $\omega=\omega_{\text {final }}$ that was chosen to be (b)

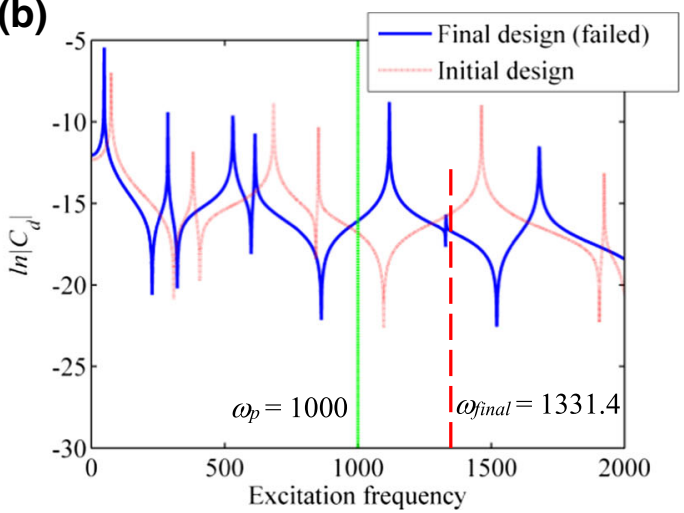

i.e. $\omega \in\left[\Omega_{6}, \Omega_{7}\right]$. (b) Comparison of the dynamic compliance curves for the initial design (Fig. 14b) and the final reference design (Fig. 21a). The excitation frequency cannot reach the prescribed value $\omega_{p}=1000$, which implies that the solution set of the design Case $\mathrm{C} 2$ is a null set

slightly larger than zero. The result was that for $\omega$ tending to zero, both the fundamental resonance frequency and the dynamic compliance of the design tend to zero, while the static compliance and hence the static displacements of the design, increase very drastically at $\omega=0$. The physical interpretation of this behaviour clearly indicates that a disintegration is created in the structure in the limit of $\omega=0$, and that the vanishing of the fundamental resonance frequency must be associated with a rigid body vibration mode with infinite displacements of the design.

A common way of avoiding this kind of 'degeneracy' of the design is to include an upper bound constraint on the static compliance in the mathematical formulation (1) of the problem of minimizing the dynamic compliance, and take the sensitivity

Table 2 Comparison of the four valid local optimized solutions obtained for the excitation frequency $\omega_{p}=1000$ by the 'generalized incremental frequency method' (GIF method)

\begin{tabular}{|c|c|c|c|c|}
\hline Case & $\begin{array}{c}\text { Dynamic compliance } \\
\text { of the initial design } \\
\left(\omega_{p}=1000\right)\end{array}$ & $\begin{array}{c}\text { Dynamic compliance } \\
\text { of the optimized } \\
\text { design }\left(\omega_{p}=1000\right)\end{array}$ & Optimized topology & $\begin{array}{c}\text { Interval of } \\
\text { resonance } \\
\text { frequencies for } \omega_{p}\end{array}$ \\
\hline A & $4.908 \mathrm{E}-8$ & $3.076 \mathrm{E}-9$ & I & $\omega_{p} \in\left[\Omega_{4}^{o p t}, \Omega_{5}^{o p t}\right]$ \\
\hline B1 & $4.908 \mathrm{E}-8$ & $1.970 \mathrm{E}-9$ & & $\omega_{p} \in\left[\Omega_{3}^{o p t}, \Omega_{4}^{o p t}\right]$ \\
\hline B2 & $4.908 \mathrm{E}-8$ & $5.168 \mathrm{E}-8$ & & $\omega_{p} \in\left[\Omega_{2}^{o p t}, \Omega_{3}^{o p t}\right]$ \\
\hline $\mathrm{C} 1$ & $4.908 \mathrm{E}-8$ & $3.161 \mathrm{E}-8$ & & $\omega_{p} \in\left[\Omega_{5}^{o p t}, \Omega_{6}^{o p t}\right]$ \\
\hline
\end{tabular}


of the static compliance into account in the sensitivity analysis. Based on the algorithm mentioned above, it is then a simple task to set-up a single, unified algorithm for handling of not only 'low' and 'high' excitation frequencies, but also singleand bi-material structures, and an upper bound constraint on the static compliance. A flow chart of this version of the GIF method is designed and shown in Fig. 4.

The IF technique and the GIF method may be applied to not only the dynamic compliance minimization problem considered in the present paper, but also to similar vibro-acoustic design problems with multiple disjointed design sub-spaces. The method may be even extended further for design optimization problems concerning multiple external excitation frequencies or excitation frequency bands.

Acknowledgments The authors were very sad to hear that Professor George Rozvany unexpectedly passed away. We will sorely miss him.

The first author is indebted to George for having benefited from very inspiring co-authorship, and we have been very close friends both personally and scientifically for more than five decades.
The second author has known George for about one decade, and is very grateful to him for very interesting discussions during international conferences and for the affordable costs to young members of ISSMO for participation in the ISSMO world congresses.

Scientists in our field will long remember George as one of the "fathers" of the area of optimum structural design. Thus, during the recent three decades, George has clearly been the intellectual and managerial leader of our optimization community. George has manifested this in terms of publishing numerous important and excellent scientific papers; founding ISSMO in 1991 and since then acting as Founder President and member of the Executive Committee of ISSMO; founding the SMO journal in 1989 and since acting as Editor-in-Chief of SMO; and organizing a substantial number of international scientific conferences in our field. All these activities have demanded an abundance of time and work for George, and we all owe him very warm and sincere thanks for that.

George Rozvany was one of the intellectual leaders of our optimization community. His death may mark the end of an era in structural optimization, but his legacy will remain a source of inspiration for generations of researchers to come.

The financial support provided by the Center of Machine Acoustics and Aalborg University is gratefully acknowledged. The research is also supported by NSFC $(11372154,90816025)$ and National Basic Research Program of China (2015CB251203).

\section{Appendix}

Table 3 Identification of the resonance frequencies of the initial design by orthogonality tests

\begin{tabular}{|c|c|c|c|}
\hline \multirow[b]{2}{*}{ Order $i$ of Eigenfrequency } & \multicolumn{3}{|c|}{ Initial design $\omega=500, C_{d}=6.842 \mathrm{E}-7$} \\
\hline & Eigenfrequency $\omega_{i}$ & Resonance frequency & Orthogonality index $\left|\mathbf{P}^{\mathbf{T}} \boldsymbol{\varphi}_{i}\right|$ \\
\hline 1 & $95.31=$ & \multirow[t]{5}{*}{$\Omega_{1}$} & 4.99E-01 \\
\hline 2 & 191.35 & & 2.77E-15 \\
\hline 3 & 191.35 & & $3.48 \mathrm{E}-15$ \\
\hline 4 & 277.65 & & $1.20 \mathrm{E}-15$ \\
\hline 5 & 335.95 & & $1.09 \mathrm{E}-13$ \\
\hline 6 & $338.00=$ & \multirow[b]{4}{*}{$6.842 \mathrm{E}-7$} & $3.03 \mathrm{E}-01$ \\
\hline 7 & 415.04 & & $1.01 \mathrm{E}-15$ \\
\hline \multirow[t]{2}{*}{8} & \multirow{2}{*}{\multicolumn{2}{|c|}{$\begin{array}{l}415.04 \\
\omega=500 \text { By solution of Eqs. }(1 \mathrm{~b}, \mathrm{c}): C_{d}=6.842 \mathrm{E}-7\end{array}$}} & \\
\hline & & & \\
\hline 9 & \multirow{2}{*}{\multicolumn{2}{|c|}{$\begin{array}{l}524.97 \\
524.97\end{array}$}} & $1.10 \mathrm{E}-15$ \\
\hline 10 & & & $1.78 \mathrm{E}-15$ \\
\hline 11 & $542.49=$ & \multirow[t]{8}{*}{$\Omega_{3}$} & 7.12E-02 \\
\hline 12 & 594.60 & & $5.07 \mathrm{E}-17$ \\
\hline 13 & 598.23 & & 7.73E-16 \\
\hline 14 & 668.69 & & $9.28 \mathrm{E}-16$ \\
\hline 15 & 668.69 & & $1.50 \mathrm{E}-15$ \\
\hline 16 & 714.16 & & $2.30 \mathrm{E}-16$ \\
\hline 17 & 714.16 & & $6.76 \mathrm{E}-16$ \\
\hline 18 & 749.32 & & $7.93 \mathrm{E}-15$ \\
\hline 19 & $750.36=$ & \multirow[t]{6}{*}{$\Omega_{4}$} & $1.96 \mathrm{E}-01$ \\
\hline 20 & 795.73 & & $1.38 \mathrm{E}-16$ \\
\hline 21 & 815.67 & & $2.24 \mathrm{E}-16$ \\
\hline 22 & 815.67 & & $3.20 \mathrm{E}-16$ \\
\hline 23 & 874.97 & & $3.40 \mathrm{E}-16$ \\
\hline 24 & 922.24 & & $3.86 \mathrm{E}-15$ \\
\hline 25 & $926.83=$ & \multirow{7}{*}{$\Omega_{5}$} & $6.39 \mathrm{E}-02$ \\
\hline 26 & 975.53 & & $2.52 \mathrm{E}-16$ \\
\hline 27 & 1006.88 & & $3.75 \mathrm{E}-11$ \\
\hline 28 & 1006.88 & & $2.06 \mathrm{E}-08$ \\
\hline 29 & 1066.38 & & $2.17 \mathrm{E}-17$ \\
\hline 30 & 1068.95 & & $1.39 \mathrm{E}-16$ \\
\hline$\ldots$ & $\ldots$ & & $\ldots$ \\
\hline
\end{tabular}

$\left(\right.$ Case $\left.0 . \omega_{\text {init }}=500 \rightarrow \omega_{\text {final }}=\omega_{p}=500\right)$ 
Table 4 Identification of the resonance frequencies of local optimized design by orthogonality tests

Local optimized design $\omega=\omega_{p}=500, C_{d}=5.013 \mathrm{E}-8$

\begin{tabular}{|c|c|c|c|}
\hline \multirow{2}{*}{ Order $i$ of Eigenfrequency } & & & \\
\hline & Eigenfrequency $\omega_{i}$ & Resonance frequency & Orthogonality index $\left|\mathbf{P}^{\mathbf{T}} \boldsymbol{\varphi}_{i}\right|$ \\
\hline 1 & $58.39=$ & $\Omega_{1}$ & 2.11E-01 \\
\hline 2 & 127.28 & & $1.94 \mathrm{E}-15$ \\
\hline 3 & 127.28 & & $1.76 \mathrm{E}-16$ \\
\hline 4 & 192.68 & & $5.75 \mathrm{E}-16$ \\
\hline 5 & 226.67 & & $2.51 \mathrm{E}-15$ \\
\hline 6 & $299.75=$ & $\Omega_{2}$ & $1.31 \mathrm{E}-01$ \\
\hline 7 & 326.49 & & $1.02 \mathrm{E}-15$ \\
\hline 8 & 326.49 & & $2.12 \mathrm{E}-15$ \\
\hline 9 & 327.50 & & $4.36 \mathrm{E}-15$ \\
\hline 10 & 327.50 & & $5.19 \mathrm{E}-15$ \\
\hline 11 & 389.06 & & $2.50 \mathrm{E}-16$ \\
\hline 12 & 426.99 & & $3.53 \mathrm{E}-16$ \\
\hline 13 & 426.99 & & $7.48 \mathrm{E}-17$ \\
\hline \multirow[t]{2}{*}{14} & 451.60 & & $3.64 \mathrm{E}-17$ \\
\hline & \multicolumn{3}{|c|}{$\omega=\omega_{p}=500$ By solution of Problem $(1 \mathrm{a}-\mathrm{e}): C_{d}=5.013 \mathrm{E}-8$} \\
\hline 15 & 530.05 & & $7.15 \mathrm{E}-16$ \\
\hline 16 & $532.59=$ & $\Omega_{3}$ & 4.16E-02 \\
\hline 17 & 560.35 & & $1.91 \mathrm{E}-16$ \\
\hline 18 & 591.67 & & $1.86 \mathrm{E}-17$ \\
\hline 19 & 622.51 & & $4.85 \mathrm{E}-16$ \\
\hline 20 & 653.30 & & $4.22 \mathrm{E}-16$ \\
\hline 21 & 653.30 & & $2.52 \mathrm{E}-16$ \\
\hline 22 & $816.22=$ & $\Omega_{4}$ & $2.98 \mathrm{E}-01$ \\
\hline 23 & 816.45 & & $4.53 \mathrm{E}-14$ \\
\hline 24 & 830.25 & & 4.47E-15 \\
\hline 25 & 830.25 & & $5.22 \mathrm{E}-15$ \\
\hline 26 & $848.99=$ & $\Omega_{5}$ & $2.78 \mathrm{E}-04$ \\
\hline 27 & 875.13 & & $8.61 \mathrm{E}-16$ \\
\hline 28 & 875.13 & & $1.41 \mathrm{E}-16$ \\
\hline 29 & 940.27 & & 7.19E-16 \\
\hline 30 & 944.51 & & $1.05 \mathrm{E}-15$ \\
\hline$\ldots$ & $\ldots$ & & $\ldots$ \\
\hline$\ldots$ & $957.72=$ & $\Omega_{6}$ & $1.44 \mathrm{E}-01$ \\
\hline
\end{tabular}

$\left(\right.$ Case A. $\left.\omega_{\text {init }}=500 \rightarrow \omega_{\text {final }}=\omega_{p}=500\right)$

Table 5 Identification of the resonance frequencies of failed local optimized design by orthogonality tests

Failed local optimized design $\omega=480.53<\omega_{p}=500, C_{d}=5.525 \mathrm{E}-5$

\begin{tabular}{llll}
\cline { 2 - 3 } Order $i$ of Eigenfrequency & Eigenfrequency $\omega_{i}$ & Resonance frequency & Orthogonality index $\left|\mathbf{P}^{\mathbf{T}} \boldsymbol{\varphi}_{i}\right|$ \\
\hline 1 & $93.69=$ & $\Omega_{1}$ & $2.40 \mathrm{E}-01$ \\
2 & 185.53 & & $3.63 \mathrm{E}-15$ \\
3 & 185.53 & $3.18 \mathrm{E}-14$ \\
4 & 303.09 & $8.56 \mathrm{E}-16$
\end{tabular}


Table 5 (continued)

Failed local optimized design $\omega=480.53<\omega_{p}=500, C_{d}=5.525 \mathrm{E}-5$

\begin{tabular}{|c|c|c|c|}
\hline \multirow{2}{*}{ Order $i$ of Eigenfrequency } & & & \\
\hline & Eigenfrequency $\omega_{i}$ & Resonance frequency & Orthogonality index $\left|\mathbf{P}^{\mathbf{T}} \boldsymbol{\varphi}_{i}\right|$ \\
\hline \multirow[t]{2}{*}{5} & 326.61 & & $2.21 \mathrm{E}-15$ \\
\hline & \multicolumn{3}{|c|}{$\omega=480.53<\omega_{p}=500$, Computed by IF technique: $C_{d}=5.525 \mathrm{E}-5$} \\
\hline 6 & $481.53=$ & $\Omega_{2}$ & 2.30E-01 \\
\hline 7 & 500.52 & & $7.26 \mathrm{E}-13$ \\
\hline 8 & 500.52 & & 5.41E-08 \\
\hline 9 & 516.53 & & $2.60 \mathrm{E}-13$ \\
\hline 10 & 516.53 & & $6.53 \mathrm{E}-13$ \\
\hline 11 & 576.93 & & $1.98 \mathrm{E}-16$ \\
\hline 12 & 615.73 & & $7.52 \mathrm{E}-16$ \\
\hline 13 & 615.73 & & $6.99 \mathrm{E}-16$ \\
\hline 14 & 672.57 & & $9.71 \mathrm{E}-15$ \\
\hline 15 & $681.98=$ & $\Omega_{3}$ & 2.19E-01 \\
\hline 16 & 708.63 & & $1.82 \mathrm{E}-16$ \\
\hline 17 & 757.36 & & $5.61 \mathrm{E}-16$ \\
\hline 18 & 762.56 & & $4.95 \mathrm{E}-16$ \\
\hline 19 & 805.82 & & $1.62 \mathrm{E}-15$ \\
\hline 20 & 805.82 & & $4.82 \mathrm{E}-15$ \\
\hline 21 & 806.73 & & $4.13 \mathrm{E}-15$ \\
\hline 22 & $831.38=$ & $\Omega_{4}$ & $1.83 \mathrm{E}-01$ \\
\hline 23 & 903.57 & & $1.52 \mathrm{E}-15$ \\
\hline 24 & 903.57 & & $9.72 \mathrm{E}-16$ \\
\hline 25 & $980.44=$ & $\Omega_{5}$ & $1.30 \mathrm{E}-01$ \\
\hline 26 & 995.01 & & $5.43 \mathrm{E}-13$ \\
\hline 27 & 1009.18 & & $2.48 \mathrm{E}-12$ \\
\hline 28 & 1028.23 & & $1.08 \mathrm{E}-11$ \\
\hline 29 & 1039.03 & & $1.18 \mathrm{E}-10$ \\
\hline 30 & 1039.03 & & $1.44 \mathrm{E}-10$ \\
\hline$\ldots$ & $\ldots$ & & $\cdots$ \\
\hline
\end{tabular}

$\left(\right.$ Case B. $\left.\omega_{\text {init }}=337.00 \rightarrow \omega_{\text {final }}=480.53<\omega_{p}=500\right)$

Table 6 Identification of the resonance frequencies of local optimized design by orthogonality tests

\begin{tabular}{|c|c|c|c|}
\hline \multirow[b]{2}{*}{ Order $i$ of Eigenfrequency } & \multicolumn{3}{|c|}{ Local optimized design $\omega=\omega_{p}=500, C_{d}=6.424 \mathrm{E}-10$} \\
\hline & Eigenfrequency $\omega_{i}$ & Resonance frequency & Orthogonality index $\left|\mathbf{P}^{\mathbf{T}} \boldsymbol{\varphi}_{i}\right|$ \\
\hline 1 & $55.45=$ & $\Omega_{1}$ & $2.06 \mathrm{E}-01$ \\
\hline 2 & 129.23 & & $1.63 \mathrm{E}-14$ \\
\hline 3 & 129.23 & & $3.45 \mathrm{E}-14$ \\
\hline 4 & 181.99 & & $3.52 \mathrm{E}-15$ \\
\hline 5 & $234.46=$ & $\Omega_{2}$ & $1.26 \mathrm{E}-01$ \\
\hline 6 & 260.60 & & $2.53 \mathrm{E}-15$ \\
\hline 7 & 292.99 & & $1.37 \mathrm{E}-14$ \\
\hline
\end{tabular}


Table 6 (continued)

Local optimized design $\omega=\omega_{p}=500, C_{d}=6.424 \mathrm{E}-10$

\begin{tabular}{|c|c|c|c|}
\hline \multirow{2}{*}{ Order $i$ of Eigenfrequency } & \\
\hline & Eigenfrequency $\omega_{i}$ & Resonance frequency & Orthogonality index $\left|\mathbf{P}^{\mathrm{T}} \boldsymbol{\varphi}_{i}\right|$ \\
\hline 8 & \multicolumn{2}{|l|}{292.99} & 4.93E-09 \\
\hline 9 & \multicolumn{2}{|l|}{328.13} & 4.64E-15 \\
\hline 10 & \multicolumn{2}{|l|}{328.13} & $3.85 \mathrm{E}-15$ \\
\hline 11 & \multicolumn{2}{|l|}{412.32} & $2.22 \mathrm{E}-12$ \\
\hline 12 & \multicolumn{2}{|l|}{412.32} & 4.24E-07 \\
\hline 13 & \multicolumn{2}{|l|}{419.02} & $1.06 \mathrm{E}-16$ \\
\hline 14 & \multicolumn{2}{|l|}{422.83} & $2.95 \mathrm{E}-16$ \\
\hline 15 & \multicolumn{2}{|l|}{433.71} & $1.51 \mathrm{E}-16$ \\
\hline \multirow[t]{2}{*}{16} & $461.99=$ & $\Omega_{3}$ & $1.57 \mathrm{E}-03$ \\
\hline & \multicolumn{3}{|c|}{$\omega=\omega_{p}=500$, Solution by IF technique: $C_{d}=6.424 \mathrm{E}-10$} \\
\hline 17 & \multicolumn{2}{|l|}{538.39} & $5.33 \mathrm{E}-17$ \\
\hline 18 & \multicolumn{2}{|l|}{596.14} & $7.70 \mathrm{E}-17$ \\
\hline 19 & \multicolumn{2}{|l|}{596.14} & $4.59 \mathrm{E}-16$ \\
\hline 20 & \multicolumn{2}{|l|}{598.64} & $6.04 \mathrm{E}-16$ \\
\hline 21 & \multicolumn{2}{|l|}{598.64} & $4.18 \mathrm{E}-16$ \\
\hline 22 & \multicolumn{2}{|l|}{622.79} & $2.16 \mathrm{E}-16$ \\
\hline 23 & \multicolumn{2}{|l|}{630.12} & $1.19 \mathrm{E}-15$ \\
\hline 24 & \multicolumn{2}{|l|}{706.94} & $4.09 \mathrm{E}-17$ \\
\hline 25 & \multicolumn{2}{|l|}{706.94} & $4.82 \mathrm{E}-16$ \\
\hline 26 & $712.45=$ & $\Omega_{4}$ & $1.95 \mathrm{E}-04$ \\
\hline 27 & \multicolumn{2}{|l|}{760.04} & $4.12 \mathrm{E}-15$ \\
\hline 28 & $771.12=$ & $\Omega_{5}$ & $2.61 \mathrm{E}-01$ \\
\hline 29 & \multicolumn{2}{|l|}{802.50} & $1.08 \mathrm{E}-16$ \\
\hline 30 & \multicolumn{2}{|l|}{806.04} & $3.33 \mathrm{E}-16$ \\
\hline$\ldots$ & \multicolumn{2}{|l|}{$\ldots$} & $\ldots$ \\
\hline
\end{tabular}

$\left(\right.$ Case C. $\left.\omega_{\text {init }}=545.00 \rightarrow \omega_{\text {final }}=\omega_{p}=500\right)$

Table 7 Identification of the resonance frequencies of local optimized design by orthogonality tests

\begin{tabular}{|c|c|c|c|}
\hline \multirow[b]{2}{*}{ Order $i$ of Eigenfrequency } & \multicolumn{3}{|c|}{ Local optimized design $\omega=\omega_{p}=500, C_{d}=3.216 \mathrm{E}-7$} \\
\hline & Eigenfrequency $\omega_{i}$ & Resonance frequency & Orthogonality index $\left|\mathbf{P}^{\mathbf{T}} \boldsymbol{\varphi}_{i}\right|$ \\
\hline 1 & $66.37=$ & $\Omega_{1}$ & $5.28 \mathrm{E}-01$ \\
\hline 2 & 130.05 & & $5.73 \mathrm{E}-14$ \\
\hline 3 & 130.05 & & $5.71 \mathrm{E}-14$ \\
\hline 4 & 208.88 & & 4.29E-15 \\
\hline 5 & $219.38=$ & $\Omega_{2}$ & $2.72 \mathrm{E}-01$ \\
\hline 6 & 225.70 & & $1.69 \mathrm{E}-15$ \\
\hline 7 & 294.46 & & $1.01 \mathrm{E}-12$ \\
\hline 8 & 294.46 & & $1.50 \mathrm{E}-08$ \\
\hline 9 & 351.67 & & $1.63 \mathrm{E}-12$ \\
\hline 10 & 351.67 & & $9.96 \mathrm{E}-12$ \\
\hline 11 & $379.68=$ & $\Omega_{3}$ & $1.03 \mathrm{E}-01$ \\
\hline
\end{tabular}


Table 7 (continued)

Local optimized design $\omega=\omega_{p}=500, C_{d}=3.216 \mathrm{E}-7$

\begin{tabular}{|c|c|c|c|}
\hline \multirow{2}{*}{ Order $i$ of Eigenfrequency } & \\
\hline & Eigenfrequency $\omega_{i}$ & Resonance frequency & Orthogonality index $\left|\mathbf{P}^{\mathbf{T}} \boldsymbol{\varphi}_{i}\right|$ \\
\hline 12 & \multicolumn{2}{|l|}{411.79} & $3.34 \mathrm{E}-16$ \\
\hline 13 & \multicolumn{2}{|l|}{428.36} & $5.40 \mathrm{E}-16$ \\
\hline 14 & \multicolumn{2}{|l|}{438.44} & $4.90 \mathrm{E}-15$ \\
\hline 15 & \multicolumn{2}{|l|}{438.44} & $4.18 \mathrm{E}-15$ \\
\hline \multirow[t]{2}{*}{16} & $479.11=$ & $\Omega_{4}$ & $2.93 \mathrm{E}-02$ \\
\hline & \multicolumn{3}{|c|}{$\omega=\omega_{p}=500$, Solution by IF technique: $C_{d}=3.216 \mathrm{E}-7$} \\
\hline 17 & \multicolumn{2}{|c|}{503.93} & $3.29 \mathrm{E}-16$ \\
\hline 18 & \multicolumn{2}{|l|}{503.93} & $2.63 \mathrm{E}-16$ \\
\hline 19 & \multicolumn{2}{|l|}{532.60} & $1.95 \mathrm{E}-16$ \\
\hline 20 & \multicolumn{2}{|l|}{581.67} & $2.97 \mathrm{E}-16$ \\
\hline 21 & \multicolumn{2}{|l|}{586.31} & $2.54 \mathrm{E}-16$ \\
\hline 22 & \multicolumn{2}{|l|}{586.31} & $8.25 \mathrm{E}-16$ \\
\hline 23 & \multicolumn{2}{|l|}{605.21} & $9.03 \mathrm{E}-17$ \\
\hline 24 & \multicolumn{2}{|l|}{615.60} & $6.28 \mathrm{E}-17$ \\
\hline 25 & \multicolumn{2}{|l|}{630.02} & $8.67 \mathrm{E}-16$ \\
\hline 26 & \multicolumn{2}{|l|}{630.02} & $7.69 \mathrm{E}-16$ \\
\hline 27 & \multicolumn{2}{|l|}{668.62} & $4.46 \mathrm{E}-16$ \\
\hline 28 & \multicolumn{2}{|l|}{695.83} & $3.58 \mathrm{E}-14$ \\
\hline 29 & \multicolumn{2}{|l|}{695.83} & $3.84 \mathrm{E}-14$ \\
\hline 30 & \multicolumn{2}{|l|}{710.89} & $5.48 \mathrm{E}-13$ \\
\hline$\cdots$ & \multicolumn{2}{|l|}{$\ldots$} & $\ldots$ \\
\hline$\ldots$ & $722.32=$ & $\Omega_{5}$ & $2.75 \mathrm{E}-02$ \\
\hline
\end{tabular}

$\left(\right.$ Case D. $\left.\omega_{\text {init }}=752.37 \rightarrow \omega_{\text {final }}=\omega_{p}=500\right)$

Open Access This article is distributed under the terms of the Creative Commons Attribution 4.0 International License (http:// creativecommons.org/licenses/by/4.0/), which permits unrestricted use, distribution, and reproduction in any medium, provided you give appropriate credit to the original author(s) and the source, provide a link to the Creative Commons license, and indicate if changes were made.

\section{References}

Allaire G, Francfort GA (1993) A numerical algorithm for topology and shape optimization. In: Topology optimization of structures. Kluwer, Boston (Eds Bendsøe MP, Soares MCA), pp 239-248

Bendsøe MP (1989) Optimal shape design as a material distribution problem. Struct Optim 1:193-202

Bendsøe MP, Kikuchi N (1988) Generating optimal topologies in structural design using a homogenization method. Comput Methods Appl Mech Eng 71(2):197-224

Bendsøe MP, Sigmund O (1999) Material interpolation schemes in topology optimization. Arch Appl Mech 69:635-654

Bendsøe MP, Sigmund O (2003) Topology optimization: theory, methods and applications. Springer, Berlin, Heidelberg, New York

Bendsøe MP, Olhoff N, Sigmund O (eds.) (2006) Proc. IUTAM symp. topological design optimization of structures, machines and materials - status and perspectives. Copenhagen, Denmark, October 2629, 2005. Springer, Dordrecht
Bourdin B (2001) Filters in topology optimization. Int J Numer Methods Eng 50(9):2143-2158

Bruns TE, Tortorelli DA (2001) Topology optimization of non-linear elastic structures and compliant mechanisms. Comput Methods Appl Mech Eng 190(26-27):3443-3459

Calvel S, Mongeau M (2007) Black-box structural optimization of a mechanical component. Comput Ind Eng 53(3):514-530

Cheng G, Olhoff N (1982) Regularized formulation for optimal design of axisymmetric plates. Int J Solids Struct 18:153-169

Deaton JD, Grandhi RV (2014) A survey of structural and multidisciplinary continuum topology optimization: post 2000. Struct Multidisc Optim 49:1-38

Du J, Olhoff N (2004a) Topological optimization of continuum structures with design-dependent surface loading - part I: new computational approach for 2D problems. Struct Multidisc Optim 27:151-165

Du J, Olhoff N (2004b) Topological optimization of continuum structures with design-dependent surface loading - part II: algorithm and examples for 3D problems. Struct Multidisc Optim 27:166-177

Du J, Olhoff N (2007a) Topological design of freely vibrating continuum structures for maximum values of simple and multiple eigenfrequencies and frequency gaps. Struct Multidisc Optim 34(6):545, Editors's Erratum

Du J, Olhoff N (2007b) Minimization of sound radiation from vibrating bi-material structures using topology optimization. Struct Multidisc Optim 33:305-321

Du J, Olhoff N (2007c) Topological design of freely vibrating continuum structures for maximum values of simple and multiple 
eigenfrequencies and frequency gaps. Struct Multidisc Optim 34: 91-110

Du J, Olhoff N (2010) Topological design of vibrating structures with respect to optimum sound pressure characteristics in a surrounding acoustic medium. Struct Multidisc Optim 42(1):43-54

Du J, Yang R (2015) Vibro-acoustic design of plate using bi-material microstructural topology optimization. J Mech Sci Technol 29(4): 1413-1419

Du J, Song X, Olhoff N (2011) Topological design of acoustic structure based on the BEM-FEM format and the mixed formulation. Proc 9th World Congress Struct Multidisciplin Optimiz (WCSMO-9), June 13-17, 2011, Shizuoka, Japan

Eschenauer HA, Olhoff N (2001) Topology optimization of continuum structures: a review. Appl Mech Rev 54(4):331-389

Halkjær S, Sigmund O, Jensen JS (2006) Maximizing band gaps in plate structures. Struct Multidisc Optim 32:263-275

Hammer VB, Olhoff N (1999) Topology optimization with design dependent loads. Proc. WCSMO-3, third world congress of structural and multidisciplinary optimization, May 17-21, 1999, Buffalo, NY

Hammer VB, Olhoff N (2000) Topology optimization of continuum structures subjected to pressure loading. Struct Multidisc Optim 19:85-92

Jensen JS (2003) Phononic band gaps and vibrations in one- and twodimensional mass-spring structures. J Sound Vibrat 266:1053-1078

Jensen JS (2007) Topology optimization of dynamic problems with Padé approximants. Int J Numer Meth Eng 72:1605-1630

Jensen JS, Pedersen NL (2006) On maximal eigenfrequency separation in two-material structures: the 1D and 2D scalar cases. J Sound Vibrat 289(4-5):967-986

Jog CS (2002a) Topology design of structures subjected to periodic loading. J Sound Vibrat 253(3):687-709

Jog CS (2002b) Reducing radiated sound power by minimizing the dynamic compliance. Proc IUTAM Symp Designing for Quietness (ed. M.L. Munjal), Bangalore, India, December 12-14, 2000. Kluwer, Dordrecht

Jung J, Hyun J, Goo S, Wang S (2015) An efficient design sensitivity analysis using element energies for topology optimization of a frequency response problem. Comput Methods Appl Mech Eng 296: 196-210

Kang B-S, Park G-J, Arora JS (2006) A review of optimization of structures subjected to transient loads. Struct Multidisc Optim 31:81-95

Kang Z, Zhang X, Jiang S, Cheng G (2012) On topology optimization of damping layer in shell structures under harmonic excitations. Struct Multidisc Optim 46(1):51-67

Liu H, Zhang WH, Gao T (2015) A comparative study of dynamic analysis methods for structural topology optimization under harmonic force excitations. Struct Multidisc Optim 51(6):1321-1333

Ma ZD, Kikuchi N, Cheng HC (1995) Topological design for vibrating structures. Comput Methods Appl Mech Eng 121:259-280

Min S, Kikuchi N, Park YC, Kim S, Chang S (1999) Optimal topology design of structures under dynamic loads. Struct Optim 17:208-218

Nandy AK, Jog CS (2011) Optimization of vibrating structures to reduce radiated noise. Struct Multidisc Optim 45(5):717-728

Niu B, Olhoff N, Lund E, Cheng G (2010) Discrete material optimization of vibrating laminated composite plates for minimum sound radiation. Int J Solids Struct 47:2097-2114

Olhoff N, Du J (2005) Topology optimization of structures against vibration and noise. Proc 12th Int Congress Sound Vibration, ICSV12 (eds. J.L. Bento Coelho and D. Alarcao), Lisbon, Portugal, July 1114,2005

Olhoff N, Du J (2006) Topology optimization of vibrating bi-material structures with respect to sound radiation. In: Bendsøe MP, Olhoff N, Sigmund O (eds) Proc IUTAM symp. Topological design optimization of structures, machines and materials - status and perspectives. Springer, Copenhagen, pp 43-52
Olhoff N, Niu B (2015) Minimizing the vibrational response of a lightweight building by topology and volume optimization of a base plate for excitatory machinery. Struct Multidisc Optim (published online, doi :10.1007/s00158-015-1345-6)

Olhoff N, Niu B, Cheng G (2012) Optimum design of band-gap beam structures. Int J Solids Struct 49(22):3158-3169

Pedersen NL (2000) Maximization of eigenvalues using topology optimization. Struct Multidisc Optim 20:2-11

Rozvany G, Lewinski T (eds.) (2013) Topology optimization in structural and continuum mechanics. CISM, Int. Centre for Mechanical Sciences, Udine, Italy, June 18-22, 2012. Springer, Vienna

Rozvany G, Zhou M (1991) Applications of the COC method in layout optimization. In: Eschenauer H, Mattheck C, Olhoff N (eds) Proc. Int. Conf. on engineering optimization in design processes, Karlsruhe 1990. Springer, Berlin, Heidelberg, New York, pp 59-70

Rozvany G, Zhou M, Birker T (1992) Generalized shape optimization without homogenization. Struct Optim 4:250-252

Shu L, Wang MY, Fang Z, Ma Z, Wei P (2011) Level set based structural topology optimization for minimizing frequency response. J Sound Vibrat 330(24):5820-5834

Sigmund O (1997) On the design of compliant mechanisms using topology optimization. Mech Struct Mach 25:493-524

Sigmund O (2001) Microstructural design of elastic band gap structures. Proc. 4th World Congress of Structrual and Multidisciplinary Optimization, WCSMO4 (eds. G Cheng and Y Gu), Dalian, China, June 4-8, 2001. Liaoning Electronic Press: Dalian, China

Sigmund O (2007) Morphology-based black and white filters for topology optimization. Struct Multidiscip Optim 33(4-5):401-424

Sigmund O, Jensen JS (2003) Systematic design of phononic band-gap materials and structures by topology optimization. Philosophical Trans Royal Soc London, Ser A Math, Phys Eng Sci 361:10011019

Sigmund O, Maute K (2013) Topology optimization approaches A comparative review. Struct Multidisc Optim 48:1031-1055

Sigmund O, Petersson J (1998) Numerical instabilities in topology optimization: a survey of procedures dealing with checkerboards, meshdependencies and local minima. Struct Optim 16:68-75

Svanberg K (1987) The method of moving asymptotes - a new method for structural optimization. Int J Numer Meth Eng 24:359-373

Tcherniak D (2002) Topology optimization of resonating structures using SIMP method. Int J Numer Meth Eng 54:1605-1622

Tortorelli D, Michaleris P (1994) Design sensitivity analysis: overview and review. Inverse Prob Eng 1:71-105

Wilson EL, Taylor RL, Doherty WP, Ghaboussi J (1973) Incompatible displacement models. In: Fenves SJ (ed) Numerical and computer methods in structural mechanics. Academic, NewYork

Xu B, Jiang JS, Xie YM (2015) Concurrent design of composite macrostructure and multi-phase material microstructure for minimum dynamic compliance. Comput Methods Appl Mech Eng 281:200-219

Yang R, Du J (2012) Microstructural topology optimization with respect to sound power radiation. Struct Multidisc Optim 47(2):191-206

Yang X, Li Y (2013) Topology optimization to minimize the dynamic compliance of a bi-material plate in a thermal environment. Struct Multidisc Optim 47(3):399-408

Yoon GH (2010) Structural topology optimization for frequency response problem using model reduction schemes. Comput Methods Appl Mech Eng 199(25-28):1744-1763

Zhang X, Kang Z (2014) Dynamic topology optimization of piezoelectric structures with active control for reducing transient response. Comput Methods Appl Mech Eng 281:200-219

Zhao JP, Wang CJ (2015) Dynamic response topology optimization in the time domain using model reduction method. Struct Multidisc Optim. doi:10.1007/s00158-015-1328-7 